\title{
Millimeter to X-ray flares from Sagittarius $A^{*}$
}

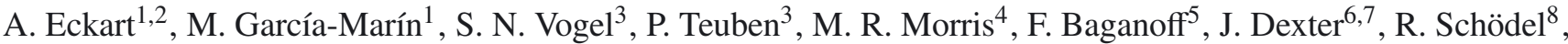 \\ G. Witzel ${ }^{1}$, M. Valencia-S. ${ }^{1,2}$, V. Karas ${ }^{9}$, D. Kunneriath ${ }^{9}$, C. Straubmeier ${ }^{1}$, L. Moser ${ }^{1}$, N. Sabha ${ }^{1}$, R. Buchholz ${ }^{1}$, \\ M. Zamaninasab ${ }^{2}$, K. Mužić ${ }^{10}$, J. Moultaka ${ }^{11}$, and J. A. Zensus ${ }^{2}$ \\ 1 I. Physikalisches Institut, Universität zu Köln, Zülpicher Str. 77, 50937 Köln, Germany \\ e-mail: eckart@ph1.uni-koeln.de \\ 2 Max-Planck-Institut für Radioastronomie, Auf dem Hügel 69, 53121 Bonn, Germany \\ 3 Department of Astronomy, University of Maryland, College Park, MD 20742-2421, USA \\ ${ }^{4}$ Department of Physics and Astronomy, University of California, Los Angeles, CA 90095-1547, USA \\ 5 Massachusetts Institute of Technology, Kavli Institute for Astrophysics and Space Research, USA \\ 6 Department of Physics, University of Washington, Seattle, WA 98195-1560, USA \\ 7 Theoretical Astrophysics Center and Department of Astronomy, University of California, Berkeley, CA 94720-3411, USA \\ ${ }^{8}$ Instituto de Astrofísica de Andalucía (CSIC), Glorieta de la Astronomía s/n, 18008 Granada, Spain \\ 9 Astronomical Institute AV CR, Czech Republic \\ 10 Department of Astronomy \& Astrophysics, University of Toronto, 50 St. George Street, Toronto, ON M5S 3H4, Canada \\ 11 IRAP, Université de Toulouse, CNRS, 14 Avenue Edouard Belin, 31400 Toulouse, France
}

Received 28 July 2011 / Accepted 24 October 2011

\begin{abstract}
Context. We report on new simultaneous observations and modeling of the millimeter, near-infrared, and X-ray flare emission of the source Sagittarius A* $\left(\mathrm{SgrA}^{*}\right)$ associated with the super-massive $\left(4 \times 10^{6} M_{\odot}\right)$ black hole at the Galactic center.

Aims. We study the applicability of the adiabatic synchrotron source expansion model and study physical processes giving rise to the variable emission of $\mathrm{SgrA}^{*}$ from the radio to the X-ray domain.

Methods. Our observations were carried out on 18 May 2009 using the NACO adaptive optics (AO) instrument at the European Southern Observatory's Very Large Telescope, the ACIS-I instrument aboard the Chandra X-ray Observatory, the LABOCA bolometer at the Atacama Pathfinder EXperiment (APEX), and the CARMA mm telescope array at Cedar Flat, California.

Results. The X-ray flare had an excess $2-8 \mathrm{keV}$ luminosity between 6 and $12 \times 10^{33} \mathrm{erg} \mathrm{s}^{-1}$. The observations reveal flaring activity in all wavelength bands that can be modeled as the signal from an adiabatically expanding synchrotron self-Compton (SSC) component. Modeling of the light curves shows that the sub-mm follows the NIR emission with a delay of about three-quarters of an hour with an expansion velocity of about $v_{\exp } \sim 0.009 \mathrm{c}$. We find source component sizes of around one Schwarzschild radius, flux densities of a few Janskys, and spectral indices $\alpha$ of about $+1\left(S(v) \propto v^{-\alpha}\right)$. At the start of the flare, the spectra of the two main components peak just short of $1 \mathrm{THz}$. To statistically explain the observed variability of the (sub-)mm spectrum of SgrA*, we use a sample of simultaneous NIR/X-ray flare peaks and model the flares using a synchrotron and SSC mechanism.

Conclusions. These parameters suggest that either the adiabatically expanding source components have a bulk motion larger than $v_{\text {exp }}$ or the expanding material contributes to a corona or disk, confined to the immediate surroundings of SgrA*. For the bulk of the synchrotron and SSC models, we find synchrotron turnover frequencies in the range of 300-400 GHz. For the pure synchrotron models, this results in densities of relativistic particles of the order of $10^{6.5} \mathrm{~cm}^{-3}$ and for the SSC models, the median densities are about one order of magnitude higher. However, to obtain a realistic description of the frequency-dependent variability amplitude of SgrA*, models with higher turnover frequencies and even higher densities are required.
\end{abstract}

Key words. accretion, accretion disks - black hole physics - Galaxy: center - Galaxy: nucleus

\section{Introduction}

Owing its proximity, Sagittarius A* (SgrA*; Balick \& Brown 1974), the compact radio source at the Galactic center, is the most intensively studied super-massive black hole (SMBH) candidate. Source structure on event-horizon scales has been detected by Doeleman et al. (2008) and Fish et al. (2011) by means of very long baseline interferometry (VLBI) at $1.3 \mathrm{~mm}$ wavelength. In the radio and millimeter wavelength regime, SgrA* is found to be variable (Zhao et al. 2003; Mauerhan et al. 2005; Eckart et al. 2008; Marrone et al. 2008; Li et al. 2009; Yusef-Zadeh et al. 2008, 2009). Its spectral energy distribution (SED) shows an inverted spectrum from the radio to the (sub-)millimeter domain (Falcke et al. 2000). It displays order-of-magnitude flares in the infrared and X-ray domain (Baganoff et al. 2001; Genzel et al. 2003; Eckart et al. 2004, 2006a; Ghez et al. 2004). Baganoff et al. (2003) detected a very low quiescent X-ray luminosity. At infrared wavelengths, the possible presence of a quiescent state is currently under investigation (see discussion in Schödel et al. 2011; Do et al. 2009; Sabha et al. 2010; Dodds-Eden et al. 2010). The sub$\mathrm{mm}$ spectrum of SgrA* itself is not very well-known owing to resolution effects and the contaminating flux density contributions from the surrounding "mini-spiral" HII region and the circumnuclear disk (CND). However, the so-called sub-millimeter bump is often attributed to SgrA* and is thought to be due to 
relativistic, thermal electrons in the innermost section of a hot, thick, advection-dominated accretion flow (Dexter et al. 2010; Narayan et al. 1995; Yuan et al. 2003). Here we refer to the submillimeter bump as the major portion of the low flux density state of $\mathrm{SgrA}^{*}$ at sub-millimeter wavelengths. It is currently unclear whether the radio spectrum is produced by a small amount of energy being injected into non-thermal electrons in the accretion flow (Yuan et al. 2003), or due to a short, mildly relativistic, optically thick jet (Falcke \& Markoff 2000) or a combination of both. A jet may have a very low surface brightness. The combination with severe foreground scattering at $\mathrm{cm}$-wavelengths has made it inaccessible to observations until now (Markoff 2005; Markoff et al. 2007). The weak quiescent X-ray emission is dominated by bremsstrahlung from cooler electrons near the Bondi radius.

Our results demonstrate that the lack of an imaged jet in SgrA* is not necessarily due to its absence, but might rather be caused by a very compact, weak source, combined with extreme scatter broadening by Galactic electrons, and the limitations of our spatial resolution, especially in the north-south direction (Markoff et al. 2007).

The highly variable radio to X-ray emission is most likely due to synchrotron and/or synchrotron self-Compton (SSC) radiation. Broderick \& Loeb (2006) and Eckart et al. (2006b) modeled the highly polarized infrared flare emission assuming orbiting inhomogeneities (i.e., hot spots) in the inner radii of the accretion flow or a temporary accretion disk.

In addition to either spiral arm models (Karas et al. 2007; Falanga et al. 2007) or jet/jet-base models (Falcke \& Markoff 2000, 2001; Markoff 2005; Markoff et al. 2007), recent attempts to explain the emission from the compact region around SgrA* have been based on a hot-spot/disk model (Eckart et al. 2006b; Broderick \& Loeb 2006), multi-spot models, or even evolving hot-spot models (Eckart et al. 2008; Yuan et al. 2009; see also Pecháček et al. 2008; Zamaninasab et al. 2011).

While hot spot models are well-suited to describe a number of observed flare features, neither the nature nor the way in which these spots can be created are well-understood (however see Yuan et al. 2009). These spots may originate as random variations resulting from magnetic turbulence inside a magnetohydrodynamic (MHD) flow (Balbus \& Hawley 1991; Armitage \& Reynolds 2003), as vortices, flux tubes, or possibly magnetic flares (Abramowicz et al. 1992; Poutanen \& Fabian 1999; Zycki 2002). Yuan et al. (2009) explain the formation of hot spots in the disk that are similar to solar spots followed by a coronal mass ejection. Several authors (e.g. Schnittman et al. 2006; Pecháček et al. 2008; Eckart et al. 2006b; Zamaninasab et al. 2010) have shown that a multi-component hot spot scenario can reproduce the observed flare emission and the overall behavior of the observed SED.

In addition multi-wavelength observations of flares suggest an adiabatically expanding blob model (Eckart et al. 2006a, 2008; Yusef-Zadeh et al. 2006a, 2008, 2009), which can explain the observed time lags between the infrared/X-ray and millimeter flares. However, a general model that provides a quantitative explanation of the frequency-dependent variability of SgrA* in the millimeter and sub-millimeter wavelengths, including the contribution of thermal electrons and relativistic electrons that result in NIR/X-ray flares, is not yet available (see comments in Dexter et al. 2010).

In this paper, we present another example of a simultaneous (sub-)mm, NIR, and X-ray measurement that allows a description in the framework of an adiabatic expansion model. The data are presented in Sect. 2 and the modeling in Sect. 3. In
Sect. 4 and especially in Sect. 4.2.2, we then use all X-ray flares that show a simultaneous (within less than about $10 \mathrm{~min}$ ) nearinfrared emission peak. We assume that the SSC process is the dominant mechanism for explaining the flares. In this case, we can use the SSC formalism in combination with the flux densities observed at different frequencies to visualize and compare different classes of flare model solutions. Limits on the source size $\theta$, magnetic field $B$, and the turn-over peak flux density $S_{m}$ of the flare then allow us to derive possible ranges for the cutoff frequency $v_{m}$ at which the emitting synchrotron component becomes optically thick. In Sect. 4.3, we then use this formalism to describe the frequency-dependent variability of SgrA* and to place further constraints especially on the magnetic field and the source size. Finally, in Sect. 5, we summarize and discuss the results of our study, and present our conclusions in Sect. 6.

\section{Observations and data reduction}

\subsection{The NIR observations}

Near-infrared observations were carried out with the NIR camera CONICA and the adaptive optics (AO) module NAOS (briefly "NACO") at the $8.2 \mathrm{~m} \mathrm{ESO} \mathrm{VLT}{ }^{1}$ unit telescope 4 (YEPUN) on Paranal, Chile, on the night of 18 May, 2009. For all observations, the infrared wavefront sensor of NAOS was used to lock the AO loop on the NIR bright ( $K$ s-band magnitude $~ 6.5$ ) supergiant IRS 7, located about 5.6" north of SgrA*. The pixel scale was 13.27 mas. Both the AO correction and the atmospheric conditions were stable during the observations. The seeing at the telescope measured in the optical was better than $\sim 0.6^{\prime \prime}$. We used an integration time of DIT $=10 \mathrm{~s}$ and a number of integrated images of NDIT $=4$. Details of the start and stop times are listed in Table 1.

All observations in the $K$ s-band $(2.2 \mu \mathrm{m})$ were dithered. The sky background was extracted from the median of stacks of dithered exposures of a dark cloud - a region practically empty of stars - about $400^{\prime \prime}$ north and $713^{\prime \prime}$ west of the target. Standard data reduction was applied (see, e.g., Eckart et al. 2006a). The images were stacked and mosaicked, the point spread functions (PSF) were extracted with StarFinder (Diolaiti et al. 2000) for deconvolution with the Lucy-Richardson (LR; Lucy 1974) algorithm.

The flux densities were measured by aperture photometry with circular apertures of 40 mas radius and corrected for extinction, using $A_{K}=2.46$ (Scoville et al. 2003; Schödel et al. 2007, 2011; Buchholz et al. 2009). Possible uncertainties in the extinction of a few tenths of a magnitude do not influence the general results obtained in this paper.

The flux density calibration was carried out using zero points for the corresponding camera setup and by comparing with the known $K$ s-band flux densities of IRS16C and IRS16NE (from e.g. Blum et al. 1996), after taking into account a correction to the $A_{K}=2.46$ value that we use here (Schödel et al. 2010). Relative photometry for SgrA* was derived using data for ten sources within 1.' 6 of SgrA* as secondary calibrators (S67, S92, S35, S8, S76, S1, S2, S87, S65, S30; Gillessen et al. 2006). The background flux in the immediate vicinity of $\mathrm{SgrA}^{*}$ was obtained by averaging the measurements at six random locations in a field located about 0. ' 6 west of SgrA* that is free of obvious stellar sources. However, the unresolved stellar background may

1 Based on observations at the Very Large Telescope (VLT) of the European Southern Observatory (ESO) on Paranal in Chile; Program: 271.B-5019(A). 
Table 1. Observational log.

\begin{tabular}{|c|c|c|c|c|}
\hline $\begin{array}{l}\text { Telescope } \\
\text { Observing ID }\end{array}$ & Instrument & band & $\begin{array}{l}\text { UT and JD } \\
\text { Start Time }\end{array}$ & $\begin{array}{l}\text { UT and JD } \\
\text { Stop Time }\end{array}$ \\
\hline CARMA & - & $100 \mathrm{GHz}$ & $\begin{array}{c}200918 \text { May } 07: 40: 20 \\
\text { JD } 2454969.819676\end{array}$ & $\begin{array}{c}18 \text { May } 12: 13: 23 \\
\text { JD } 2454970.009294\end{array}$ \\
\hline APEX & LABOCA & $345 \mathrm{GHz}$ & $\begin{array}{c}200918 \text { May } 03: 19: 00 \\
\text { JD } 2454969.638194\end{array}$ & $\begin{array}{l}18 \text { May 12:05:00 } \\
\text { JD } 2454970.003472\end{array}$ \\
\hline VLT UT 4 & NACO & $2.2 \mu \mathrm{m}$ & $\begin{array}{c}200918 \text { May } 04: 37: 55 \\
\text { JD } 2454969.692998\end{array}$ & $\begin{array}{c}18 \text { May 10:19:54 } \\
\text { JD } 2454969.930486\end{array}$ \\
\hline Chandra & ACIS-I & $2-8 \mathrm{keV}$ & $\begin{array}{c}200918 \text { May 06:40:00 } \\
\text { JD } 2454969.777778\end{array}$ & $\begin{array}{c}18 \text { May } 12: 14: 00 \\
\text { JD } 2454970.009722\end{array}$ \\
\hline Chandra & ACIS-I & $2-8 \mathrm{keV}$ & $\begin{array}{c}200918 \text { May } 02: 44: 44 \\
\text { JD } 2454969.777778\end{array}$ & $\begin{array}{l}19 \text { May 10:19:50 } \\
\text { JD } 2454970.930440\end{array}$ \\
\hline
\end{tabular}

still peak sharply at SgrA* within a distance of $0.6^{\prime \prime}$ (see, e.g. Sabha et al. 2010).

\subsection{The Chandra $X$-ray observations}

The X-ray observations of SgrA* were obtained with Chandra using the imaging array of the Advanced CCD Imaging Spectrometer (ACIS-I; Weisskopf et al. 2002) on 18-19 July 2009 (UT). The start and stop times are listed in Table 1. The instrument was operated in timed exposure mode with detectors I0-3 turned on. The time between the acquisition of different CCD frames was $3.141 \mathrm{~s}$. The event data were telemetered in faint format.

The data were reduced and analyzed using CIAO v4.1.2 software with Chandra CALDB v3.5.3 $3^{3}$. Following Baganoff et al. (2003), the level 1 data was reprocessed to remove the $0.25^{\prime \prime}$ randomization of event positions applied during standard pipeline processing and to retain events flagged as possible cosmic-ray after-glows, since the strong diffuse emission in the Galactic center causes the algorithm to flag a significant fraction of the genuine X-rays. The data were then filtered according to the standard ASCA grades. The background was stable throughout the observation, and there were no gaps in the telemetry.

The counts were extracted within radii of $0.5^{\prime \prime}, 1.0^{\prime \prime}$, and $1.5^{\prime \prime}$ around SgrA* in the $2-8 \mathrm{keV}$ band. Background counts were extracted from an annulus around $\mathrm{SgrA}^{*}$ with inner and outer radii of $2^{\prime \prime}$ and $10^{\prime \prime}$, respectively, excluding regions around discrete sources and bright structures (Baganoff et al. 2003). We note that the mean source count rates that we obtained during the quiescent source state are consistent with the same count rates of previous observations (e.g., Baganoff et al. 2001, 2003).

\subsection{The millimeter data}

We also present observations of SgrA* using CARMA ${ }^{4}$. We observed the Galactic center on 18 May 2009 at $100 \mathrm{GHz}$. CARMA (Combined Array for Research in mm-wave Astronomy) is

\footnotetext{
${ }^{2}$ Chandra Interactive Analysis of Observations (CIAO), http://cxc.harvard.edu/ciao.

${ }^{3}$ http://cxc.harvard.edu/caldb

4 Support for CARMA construction was derived from the states of California, Illinois, and Maryland, the Gordon and Betty Moore Foundation, the Kenneth T. and Eileen L. Norris Foundation, the Associates of the California Institute of Technology, and the National Science Foundation. Ongoing CARMA development and operations are supported by the National Science Foundation under a cooperative agreement, and by the CARMA partner universities.
}

located in Cedar Flat, Eastern California, and consists of 15 antennas $(6 \times 10.4 \mathrm{~m}$ and $9 \times 6.1 \mathrm{~m})$. The interferometer data were reduced using the Miriad interferometric data reduction package. Details of the observation are given in Table 1. Details of the data reduction are given in Kunneriath et al. (2010).

\subsection{The sub-millimeter data}

The May 2009 Galactic center $870 \mu \mathrm{m}$ data were taken with the LABOCA bolometer array, located on the Atacama Pathfinder EXperiment (APEX) telescope at the Llano Chajnantor. The radiation is fed through conical horns to the detectors of the Large APEX BOlometer CAmera (LABOCA), an array of 295 composite bolometers that are extremely sensitive to continuum radiation. With a total bandwidth of about $60 \mathrm{GHz}$, the system is optimized for the $345 \mathrm{GHz}$ atmospheric window. A detailed description of the LABOCA data recording and analysis in the context of SgrA* is given by e.g., Eckart et al. (2008) and Garcia-Marin et al. (2011a,b). For completeness, we summarize some of the essentials that are relevant to this paper.

The full width half maximum (FWHM) of the point spread function (PSF) at $870 \mu \mathrm{m}$ is $\sim 19^{\prime \prime}$. We used on-the-fly (OTF) maps perpendicular to the Galactic plane (Weiss et al. 2008; Siringo et al. 2007) to allow for the optimal reconstruction of the complex sub-mm emission in the GC region. The maps were observed with a scanning speed of $3^{\prime} /$ s modulating the source signals even for extended structures into the useful post-detection frequency band of LABOCA $(0.1-12.5 \mathrm{~Hz})$. Scanning artefacts in the reconstruction of the extended thermal emission surrounding $\mathrm{SgrA}^{*}$ were avoided by acquiring and averaging maps from the data with an inclination of $-10^{\circ}, 0^{\circ}$, and $+10^{\circ}$ with respect to an axis orthogonal to the Galactic plane. The mapping steps perpendicular to the scanning direction was $30^{\prime \prime}$ and the integration time $280 \mathrm{~s}$, yielding an rms noise level of $\sim 150 \mathrm{mJy} / \mathrm{beam}$ for each map.

Either G10.62 or IRAS16293-2422 was observed as a secondary calibrator source immediately after each Galactic center map. We reduced the data with the $\mathrm{BoA}^{5}$ software package. The data reduction process included correction for atmospheric zenith opacity (towards the Galactic center $\tau \sim 0.3$ up to 0.7 ), flat-fielding, de-spiking, correlated sky noise removal, and the removal of additional correlated noise caused by instrumental effects. After correcting for pointing offsets between individual maps (determined from the position of SgrA* in each map) all scans were co-added to obtain high signal-to-noise ratio $(\mathrm{S} / \mathrm{N})$, $48^{\prime} \times 25^{\prime}$ fully sampled maps of the sub-mm emission in the

5 BoA: http://www.astro.uni-bonn.de/boawiki/Boa 
GC regions. From this combined map, the point source SgrA* was modeled with a Gaussian and removed. We considered the remaining image as a reference of the extended $870 \mu \mathrm{m}$ emission surrounding $\mathrm{SgrA}^{*}$, in which $\mathrm{SgrA}^{*}$ was effectively in an off state. Each data point of the SgrA* sub-mm light curve was derived from the model-subtracted maps, modeling a Gaussian source and deriving the peak. From a comparison of different reference sources, we estimated a $4 \%$ relative point-to-point uncertainty in the calibration of the light curve. A more detailed description of the data reduction and analysis of the images was given by e.g. Eckart et al. (2008) and Garcia-Marin et al. (2011a,b).

\subsection{The sample of simultaneous $N I R / X$-ray flares}

In addition to the new data presented in this paper, we used a sample of all X-ray flares that show a simultaneous (within less than about $10 \mathrm{~min}$ ) near-infrared emission peak. These events are listed in Table 3. In Sect. 5.2, we discuss the effects of the flare profile. All NIR flux densities taken from the literature were corrected to a value of $A_{K}=2.46$ (Schödel et al. 2010). For the $L^{\prime}$-band flares reported by Dodds-Eden et al. (2009; 4 April 2007) and by Trap et al. (2010; 1 April 2009), we corrected the $L^{\prime}$-band flux densites to an extinction of $A_{L}^{\prime}=1.23$ (Schödel et al. 2010) and extrapolated to the $K$ s-band assuming a spectral index of $0.7 \pm 0.3$ (Hornstein et al. 2007; see also Bremer et al. 2011). In this sample, we also included an X-ray flare that had not been detected simultaneously in the NIR (Marrone et al. 2008). In this case, we used the upper limits as the peak flare flux in the NIR. The details of the observations and data reduction for the flares in this sample are given in the listed references in Table 3. In case these peaks were part of a more complex flare structure, we provide the flare designation used in the corresponding reference.

\section{Flare analysis}

\subsection{Adiabatically expanding source components}

Our basic assumption is that some of the positive flux density deviations from a low state in the sub-mm light curves are physically linked to the NIR/X-ray flares. The model is represented by an expanding uniform blob of relativistic electrons with an energy-dependent particle number density $\rho(E) \propto E^{-p}$ threaded by a magnetic field. In the context of SgrA*, this model was explained in detail by, e.g., Eckart et al. (2006a, 2008) and Yusef-Zadeh et al. (2008, 2009). For completeness, we summarize the definition of essential quantities and features of the model. As a consequence of the adiabatic expansion, the magnetic field inside the blob declines as $R^{-2}$, the energy of relativistic particles as $R^{-1}$, and the density of particles as $R^{-3}$ (van der Laan 1966). The synchrotron optical depth at frequency $v$ then scales as

$\tau_{v}=\tau_{0}\left(\frac{v}{v_{0}}\right)^{-(p+4) / 2}\left(\frac{R}{R_{0}}\right)^{-(2 p+3)}$

and the flux density scales as

$S_{v}=S_{0}\left(\frac{v}{v_{0}}\right)^{5 / 2}\left(\frac{R}{R_{0}}\right)^{3} \frac{1-\exp \left(-\tau_{v}\right)}{1-\exp \left(-\tau_{0}\right)}$,

were $R_{0}, S_{0}$, and $\tau_{0}$ are the size, flux density, and optical depth at the peak frequency $v_{0}$ of the synchrotron spectrum. The goal of the present model is to combine the description of an adiabatically expanding cloud with a synchrotron self-Compton formalism, as this is the most likely physical scenario to explain the delay between the sub-mm and the simultaneous near-IR and X-ray peaks. Thus, we use the definition of $\tau_{0}$ as the optical depth corresponding to the frequency at which the flux density is a maximum (van der Laan 1966), rather than the definition of $\tau_{0}$ as the optical depth at which the light curve for any particular frequency peaks (Yusef-Zadeh et al. 2006b). This implies that $\tau_{0}$ depends only on $p$ by means of the condition

$e^{\tau_{0}}-\tau_{0}(p+4) / 5-1=0$,

and ranges from, e.g., 0 to 0.65 as $p$ ranges from 1 to 3 . Therefore, given the particle energy spectral index $p$ and the peak flux $S_{0}$ in the light curve at some frequency $v_{0}$, this model predicts the variation in flux density at any other frequency as a function of the expansion factor $\left(R / R_{0}\right)$.

Finally, a model for $R(t)$ is required to convert the dependence on radius into one of time: as in previous references (e.g. Yusef-Zadeh et al. 2006b; Eckart et al. 2008) we adopt a simple linear expansion at constant expansion speed $v_{\text {exp }}$, such that $R-R_{0}=v_{\exp }\left(t-t_{0}\right)$. For $t \leq t_{0}$, we have made the assumption that the source has an optical depth equal to its frequency-dependent initial value $\tau_{v}$ at $R=R_{0}$. Hence, in the optically thin part of the source spectrum, the flux initially increases with the source size at a constant $\tau_{v}$ and then decreases owing to the decreasing optical depth as a consequence of the expansion. For the $\sim 4 \times 10^{6} \mathrm{M}_{\odot}$ SMBH at the position of SgrA* (e.g. Eckart et al. 2002; Ghez et al. 2003; Schödel et al. 2002; Eisenhauer 2003; Gillessen et al. 2009), one Schwarzschild radius is $R_{\mathrm{s}}=2 \mathrm{GM} / \mathrm{c}^{2} \sim 10^{10} \mathrm{~m}$ and the velocity of light corresponds to about $100 R_{\mathrm{s}}$ per hour. For $t>t_{0}$, the decaying flank of the curve can be shifted towards later times by first, increasing the turnover frequency $v_{0}$ or the initial source size $R_{0}$, and second, by lowering the spectral index $\alpha_{\text {synch }}$ or the peak flux density $S_{0}$. Increasing the adiabatic expansion velocity $v_{\exp }$ shifts the peak of the light curve to earlier times. Adiabatic expansion also leads to a slower decay rate and a longer flare timescale at lower frequencies.

Here we assume that the radiating spots are located in or close to the disk and that the expansion takes place in that region. Yuan et al. (2009) demonstrated that spots are formed as flux ropes within the differentially rotating disk. Shortly after their formation, they very rapidly leave the disk into the corona and in a magnetic reconnection event the ropes are discharged by magnetic compression forces with mass ejections at relativistic speeds. Within the corona, the spots expand almost adiabatically (Yuan et al. 2009). They quickly become optically thin in the radio and can be regarded as blobs within the coronal plasma. It is conceivable that the spots, i.e. the flux ropes, also expand at rather slow velocities within the differential rotating disk just before the ejection. (see Fig. 3 in Eckart et al. 2011).

\subsection{An adiabatic expansion model of the May 2009 data}

In Fig. 1, we show the results of describing our May 2009 multi-frequency data using an adiabatic expansion model with parameters listed in Table 4. We show the data with red error bars connected by a thin black line. The model consists of two components that describe the earlier (component A; thick red solid line) and the later X-ray peak (component B; thick back dashed line). A third component is required to account for additional flux in the early wing of the CARMA flare (component C; thick solid blue line; see discussion in Sect. 5.3). The overall model - 

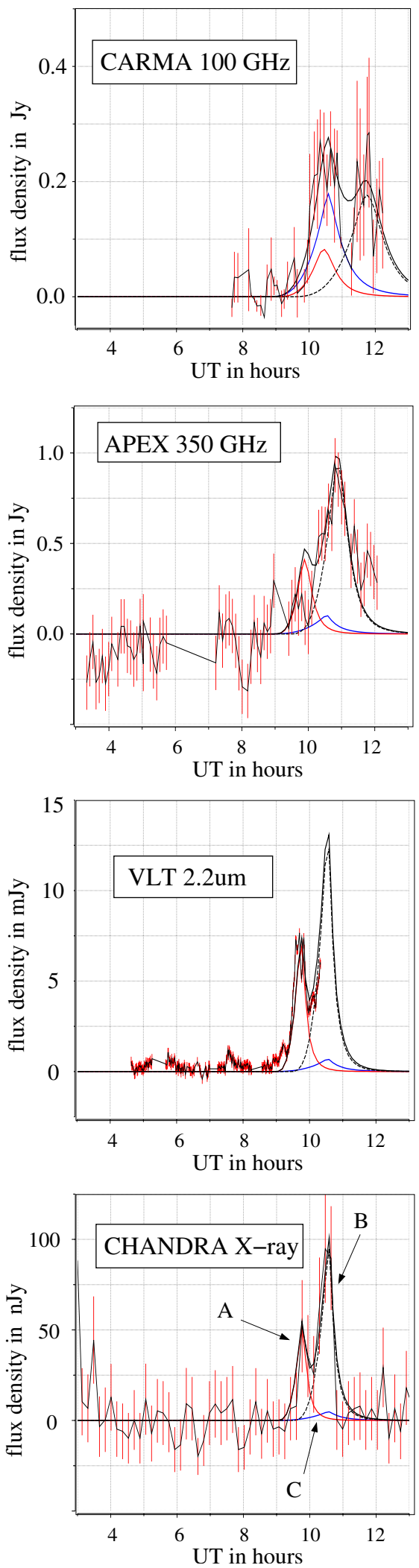

Fig. 1. An adiabatic expansion model of the May 2009 flare.

as a sum of all three components - is shown as a thick black solid line. In the framework of previously described multi-spot models or evolving hot-spot models (Eckart et al. 2008; Yuan et al. 2009; see also Pecháček et al. 2008; Zamaninasab et al. 2011), the adoption of three components is a reasonable assumption. The offset flux densities above which we attribute the flux density to the flare event are listed in Table 2. We consider the minimum flux density in a light curve (of a few hours duration) taken over at least three consecutive measurements as a flux contribution that can be attributed to a constant offset and/or to a flux density variation that is taking place over a much longer timescales than the variations considered here. In the (sub-)mm domain, the light curves taken at different epochs always provided similar lower limits to the variability modulation (see data referred to in Sect. 4.3 and discussion in Eckart et al. 2008; and Garcia-Marin 2011a,b). These offsets were subtracted from the flare profiles shown in Fig. 1. The first two components are determined by the two X-ray peaks. The sizes are constrained by the peak flux densities in the X-ray (i.e., the SSC scattering efficiency) and the flare profile widths. The peak flux density in the NIR and the (sub-)mm fluxes determines the spectral index of the optically thin part and turnover frequency of the optically thick part of the synchrotron spectrum for the first component. For the second component, the peak time and width of the $345 \mathrm{GHz}$, NIR, and X-ray flare constrains its spectral index and other quantities. At the $100 \mathrm{GHz}$ observing frequency of CARMA, we found that we need to include an additional low-frequency component to explain the flux at the time of the peak of the first component. This additional component $C$ needs to have a peak frequency close to the CARMA observing frequency. The multi-frequency data cannot be explained well in the framework of an adiabatic expansion model with fewer than three source/flare components. The typical uncertainties in the component parameters are given in the third row of Table 4. If the parameters varied by more than these quantities, there would be locations in the light curves where the deviation of the overall model from the measured data is well above three times the uncertainty in the data points.

The model results in a $11.88 \mu \mathrm{m}$ flux density of $71 \mathrm{mJy}$ that lies well below the de-reddened $3 \sigma$ upper limit at $11.88 \mu \mathrm{m}$ of $86 \mathrm{mJy}$ that includes the uncertainties in the extinction correction (Dodds-Eden et al. 2010; see comments by Schödel et al. 2011). This limit was obtained from measurements that are simultaneous with flare observations (Dodds-Eden et al. 2010). The spectral index of $\alpha=+0.15$ that would meet the limit, is too flat. The source size and flux densities determined by the (sub-)mm and NIR flare fluxes and profiles correspond to far too high X-ray fluxes. Even a spectral index of $\alpha=+0.93$, which would infer a flux only $3 \sigma$ above the limit, implies sizes and turnover frequencies that result in too broad flare profiles. A solution in which the NIR and X-ray flux densities would be due to a single optically thin synchrotron spectrum gives a spectral index of $\alpha_{\mathrm{NIR}, \mathrm{X} \text {-ray }}=+1.63$ and strongly violates the assumed MIR flux density limit.

\section{Placing limits on the SgrA* source properties}

Here we summarize the limits that can be put on SgrA* source parameters. In the following sections, these limits will be used to constrain the radiation mechanisms and to describe the variabilty across the millimeter and sub-millimeter spectrum.

\subsection{SgrA* source parameters}

\subsubsection{The optically thin spectral index}

Several NIR observational campaigns have been conducted that have provided a variety of spectral index measurements. A comprehensive summary is given by Trap et al. (2011) in their Table 4 (see also Hornstein et al. 2007). One of the lower spectral index measurements is $\alpha=+0.4 \pm 0.3$ presented by 
A\&A 537, A52 (2012)

Table 2. Characteristics of the flares observed by CARMA, APEX, the VLT, and Chandra.

\begin{tabular}{lcccccccc}
\hline \hline $\begin{array}{l}\text { Spectral } \\
\text { domain }\end{array}$ & Date & Flare start & Flare stop & $\begin{array}{c}\text { FWZP } \\
(\mathrm{min})\end{array}$ & $\begin{array}{c}\text { FWHM } \\
(\mathrm{min})\end{array}$ & $\begin{array}{c}\text { Aperture } \\
\text { Size } \\
(\operatorname{arcsec})\end{array}$ & $\begin{array}{c}\text { Flare } \\
\text { Offset } \\
\text { state }\end{array}$ \\
\hline $100 \mathrm{GHz}$ & 18 May 2009 & $10: 10 \pm 10 \mathrm{~min}$ & $>12: 13$ & $>150$ & $>140$ & 3 & $0.24 \pm 0.05 \mathrm{Jy}$ & $0.9 \mathrm{Jy}$ \\
$345 \mathrm{GHz}$ & 18 May 2009 & $09: 30 \pm 20 \mathrm{~min}$ & $>12: 05$ & $>150$ & $120 \pm 10$ & 19 & $0.85 \pm 0.15 \mathrm{Jy}$ & $2.9 \mathrm{Jy}$ \\
NIR & 18 May 2009 & $09: 10 \pm 10 \mathrm{~min}$ & $>10: 20$ & $>70$ & $>50$ & 0.06 & $10 \pm 0.5 \mathrm{mJy}$ & $1.0 \mathrm{mJy}$ \\
X-ray & 18 May 2009 & $09: 10 \pm 10 \mathrm{~min}$ & $11: 10 \pm 10 \mathrm{~min}$ & $100 \pm 10$ & $60 \pm 10$ & 1.50 & $40 \pm 5 \mathrm{nJy}$ & $20 \mathrm{nJy}$ \\
\hline
\end{tabular}

Notes. For all bands, we list the estimated start and stop times, the full width at zero power (FWZP) and FHWM values, as well as the peak and quiescent flux densities. The NIR refers to the $2.2 \mu \mathrm{m} K_{\mathrm{s}}$-band. The X-ray refer to the $2-8 \mathrm{keV}$ band. In the radio, the aperture size refers to the FWHM of the synthesized (CARMA) and primary beam (APEX).

Table 3. Table of X-ray flare events with NIR flux densities measured at the time of the X-ray flare peak emission.

\begin{tabular}{cccccc}
\hline \hline & $\begin{array}{c}\text { X-ray } \\
\mu \mathrm{Jy}\end{array}$ & $\begin{array}{c}\text { NIR } K \text { s-band } \\
\mathrm{mJy}\end{array}$ & Date & Designation & Reference \\
\hline 1 & $0.039 \pm 0.011$ & $2.7 \pm 0.3$ & 20 June 2003 & & $(1)$ \\
2 & $0.223 \pm 0.027$ & $4.4 \pm 1.1$ & 07 July 2004 & $\phi 3$ & $(2)$ \\
3 & $0.051 \pm 0.008$ & $5.8 \pm 0.3$ & 30 July 2005 & & $(3)$ \\
4 & $<0.016$ & $4.1 \pm 0.5$ & 31 July 2005 & & $(5)$ \\
5 & $1.750 \pm 0.200$ & $14.5 \pm 2.0$ & 04 April2007 & & $(6,7,8)$ \\
6 & $0.090 \pm 0.010$ & $7.2 \pm 1.0$ & 1 April 2009 & $\mathrm{A}$ & $(9)$ \\
7 & $0.135 \pm 0.010$ & $3.7 \pm 0.7$ & 4 April 2009 & $\mathrm{B}$ & $(9)$ \\
8 & $0.040 \pm 0.008$ & $10.2 \pm 0.5$ & 18 May 2009 & $\mathrm{A}$ & $(4)$ \\
\hline
\end{tabular}

Notes. For flare 5 and 6 , the $K$ s-band flux density was extrapolated from $L^{\prime}$-band.

References. (1) Eckart et al. (2004); (2) Eckart et al. (2006a); (3) Eckart et al. (2008); (4) this work; (5) Marrone et al. (2008); (6) Dodds-Eden et al. (2009, 2010); (7) Sabha et al. (2010); (8) Yusef-Zadeh et al. (2009); (9) Trap et al. (2011).

Table 4. Properties of the adiabatically expanding synchrotron source components.

\begin{tabular}{lcccccccccc}
\hline \hline Quantity & $S_{m}$ & $\alpha$ & $\theta$ & $v_{m}$ & $S_{\text {NIR,SYN }}$ & $S_{\text {NIR,SSC }}$ & $S_{\text {X-ray }}$ & $B$ & $t_{0}$ & $v_{\exp }$ \\
\hline Units & $\mathrm{Jy}$ & & $R_{S}$ & $\mathrm{GHz}$ & $\mathrm{mJy}$ & $\mathrm{mJy}$ & $\mathrm{nJy}$ & $\mathrm{G}$ & $\mathrm{UT} \mathrm{h}$ & $\mathrm{c}$ \\
\hline Uncertainty & $20 \%$ & 0.2 & 0.15 & $20 \%$ & $10 \%$ & $10 \%$ & $10 \%$ & $20 \%$ & 0.1 & 0.0015 \\
\hline A & 0.85 & +0.92 & 0.76 & 700 & 6.7 & 0.13 & 37 & 48 & 9.78 & 0.010 \\
B & 2.80 & +1.11 & 1.10 & 900 & 10.6 & 1.19 & 63 & 75 & 10.58 & 0.010 \\
C & 0.2 & +0.84 & 1.18 & 125 & 0.56 & $<0.01$ & $<2$ & 5 & 10.58 & 0.010 \\
\hline
\end{tabular}

Notes. Listed are: peak flux $S_{m}$, spectral index $\alpha$, sources size $\theta$, peak frequency $v_{m}$, NIR synchrotron flux $S_{\text {NIR,SYN }}$, NIR SSC flux $S_{\text {NIR,SSC, X-ray }}$ flux $S_{\mathrm{X} \text {-ray }}$, magnetic field $B$, time of birth (UT hours), and expansion velocity $v_{\exp }$. The flux density uncertainty in the C component could be higher, and is mainly constrained by the CARMA data.

Trap et al. (2011). For $S(v) \propto v^{-\alpha}$, this corresponds to a steep, optically thin spectrum. The reported values for the NIR spectral index can be rather steep. Values as low as $+4 \pm 1$ (Eisenhauer et al. 2005) or +2.6 (Krabbe et al. 2006) have been reported. However, spectral index measurements at low flux densities are difficult and it can also not be excluded that in some cases synchrotron losses significantly steepen the NIR spectra (Bremer et al. 2011; Eckart et al. 2006a,b). From several flare measurements listed in Table 4 by Trap et al. (2011), it is apparent that the overall spectral indices between both the NIR and X-ray domain can often be of the order of +1.5 . Under the assumption that the NIR and X-ray emission are due to synchrotron radiation from the same population of relativistic electrons, this would be a lower limit to an optically thin synchrotron spectral index that is not strongly affected by synchrotron losses. To cover a broad range of models for the later discussion of different synchrotron and SSC models, we adopt a range from +0.3 to +1.5 for the optically thin synchrotron spectral index.

\subsubsection{The SED in the sub-millimeter domain}

An estimate of the overall SED turnover frequency can be used as an upper limit to turnover frequencies of the bright source components that contribute mostly to flares below the SED turnover frequency. This does not exclude that weaker flare components become optically thick at frequencies above the SED turnover frequency. The SED turnover frequency can be estimated by extrapolating the spectral shapes obtained at millimeter radio and near-infrared wavelengths and calculating the corresponding crossing frequencies and flux densities.

For near-infrared flare emission, we assume a spectral index of $\alpha_{\mathrm{NIR}}=+0.7 \pm 0.3$. Hornstein et al. (2007) claimed that the spectral index is independent of flux density (see also Bremer et al. 2011). For our estimate, we also assumed a flare flux density of $10 \mathrm{mJy}$ at $2.2 \mu \mathrm{m}$. At millimeter wavelengths the spectrum of $\operatorname{SgrA}^{*}$ is inverted (i.e. has a negative spectral index with $\left.S(v) \propto v^{-\alpha}\right)$. For millimeter spectral indices of $\alpha_{\mathrm{mm}}=-0.5 \pm 0.25$ (inverted spectra) and a typical flux density 
for the variable portion of the source of $0.25 \mathrm{Jy}$ at $230 \mathrm{GHz}$, we then found that extrapolations of the two spectra cross at frequencies of between $80 \mathrm{GHz}$ and about $3 \mathrm{THz}$ at flux densities ranging between 0.2 and $1 \mathrm{Jy}$.

Since a variable and non-variable source component contribute to the flux densities at different $\mathrm{mm}$ - and sub-mm frequencies, the intrinsic millimeter spectrum of the variable source component may be even more inverted. For an optically thick synchrotron spectral index of $\alpha_{\mathrm{mm}}=-2.5$, we found crossing frequencies between about 200 and $600 \mathrm{GHz}$ with flux densities between 0.14 and $2.3 \mathrm{Jy}$.

From $345 \mathrm{GHz}$ and $690 \mathrm{GHz}$ SMA measurements, there is evidence that the overall spectrum of SgrA* peaks around $345 \mathrm{GHz}$ (Marrone 2006; Marrone et al. 2006a,b). The timeaveraged data presented by Marrone $(2006 a, b)$ result in an average $230 \mathrm{GHz}$ total flux density of $3.75 \pm 0.48 \mathrm{Jy}$ and a spectral index of $\alpha_{230 / 690 \mathrm{GHz}}=+0.18 \pm 0.34$ ( $1 \sigma$ uncertainties; Dexter et al. 2010). Dexter et al. (2010) note that the $230 \mathrm{GHz}$ flux measurements during these SMA observations were $50 \%$ higher than during the VLBI measurements from Doeleman et al. (2008, 2009). Thus, it is possible that the spectrum between $230 \mathrm{GHz}$ and $690 \mathrm{GHz}$ may actually be flatter than the time-averaged value obtained by Marrone (2006a,b) or even inverted. If so, then the suggested maximum at $345 \mathrm{GHz}$ may be rather broad, with substantial flux densities or even a turnover (of the entire SED or variable source component) at higher frequencies.

Lower mm-flux densities result in even higher (sub-)mm crossing frequencies at lower flux densities. Crossing frequencies above a given observing frequency will result in lower flux densities at later times if the synchrotron component evolves through adiabatic expansion. In the following, we consider synchrotron turnover frequencies of between $50 \mathrm{GHz}$ and $3 \mathrm{THz}$.

\subsubsection{Limits to the degree of variability}

Observations and simulations consistently indicate that at frequencies above $200 \mathrm{GHz}$ the variability amplitudes are of the order of 40\%-50\% (Garcia-Marin et al. 2011a,b; Dexter et al. 2009, 2010; Li et al. 2009; Marrone et al. 2006a,b, 2007). At lower frequencies (corresponding to wavelengths of $0.7 \mathrm{~cm}$ to $3.0 \mathrm{~cm}$ ), the observed variability amplitudes are of the order of $10 \%$ (Macquart \& Bower 2006; Bower et al. 2002; Falcke et al. 1998) or lower.

\subsubsection{Limits to the source size}

Light travel arguments allow us to place limits on the size of the emitting source. Jones et al. (1974a,b; see also Pauliny-Toth \& Kellermann 1966; van der Laan 1966) found an upper limit to the radius of a variable non-thermal source of $r=3 \beta_{0} c t$, were $\beta_{0}$ is the expansion speed of the source in units of the speed of light $c$. The interpretation of flux variabilities observed at different radio- and millimeter frequencies implies an adiabatic expansion speed of the order of $0.01 \mathrm{c}$ with typical rise times of about $0.5 \mathrm{~h}$. This results in an upper limit to the source diameter of the order of $2.7 R_{\mathrm{S}}$.

\subsubsection{The magnetic field strength $B$}

All estimates of the magnetic field strength indicate that it is of the order of a few $10 \mathrm{G}$. Yuan et al. (2004) estimate $\sim 10 \mathrm{G}$ for the case of spherical accretion. Falcke \& Markoff (2000) find a field strength of $\sim 25 \mathrm{G}$ for the foot point of a hypothetical jet. Estimates from relativistic MHD simulations result in values in the range of 30-50 G (Moscibrodzka et al. 2009; Dexter et al. 2010; Shcherbakov \& Baganoff 2010).
A recent measurement of the lower limit to the brightness temperature of the VLBI source SgrA* at $230 \mathrm{GHz}$ was $T_{\mathrm{b}}=$ $2 \times 10^{10} \mathrm{~K}$. From first principles, one can derive a relation between the self-absorption peak frequency $v_{\text {int }}$ in $\mathrm{GHz}$, the magnetic field $B$ in $\mathrm{G}$, and the equipartition brightness temperature $T_{12}$ in units of $10^{12} \mathrm{~K}$ for a synchrotron source without consideration of any bulk motion (e.g. Singal 2009):

$B=7.5 \times 10^{-5} v_{\text {int }} T_{12}^{-2}$.

With the relation between observed and intrinsic flux densities $S_{\text {obs }}=\delta^{3-\alpha} S_{\text {int }}$ and frequencies $v_{\text {obs }}=\delta v_{\text {int }}$, the brightness temperature for a synchrotron source (including the possibility of beaming) is defined as

$T_{12}=\frac{1.22 S_{\mathrm{obs}}}{\theta^{2} v_{\mathrm{obs}}^{2}}=\frac{1.22 S_{\mathrm{int}}}{\theta^{2} v_{\mathrm{int}}^{2}} \delta^{1-\alpha}$,

were $\theta$ is the source diameter in milliarcseconds. For $\delta \sim 1.2$ or less (e.g. as in the case of orbital motion; see Sect. 4.2.1) and $\alpha \sim 1$, we find that $\delta^{1-\alpha} \sim 1$. With a lower limit to the brightness temperature of $2 \times 10^{10} \mathrm{~K}$ and assuming that the observing frequency is close to the peak frequency of the source component spectrum, we obtain an upper limit to the magnetic field strength of about $50 \mathrm{G}$. For larger bulk motions, flatter optically thin spectra, or deviations from equipartition, the field strengths may be higher.

\subsubsection{The synchrotron cutoff frequency $v_{2}$}

The cutoff frequency $v_{2}$ depends on the product of electron boosting factor $\gamma_{\mathrm{e}}$ and the magnetic field strength $B$, and can be calculated as $v_{2}=2.8 \times 10^{6} B \gamma_{\mathrm{e}}^{2}$. In the following, we assume a range of possible cutoff frequencies and magnetic fields. If the cutoff frequency due to synchrotron losses is assumed to be located just short of the infrared $K$-band or between the NIR and X-ray domain, we find $\gamma_{\mathrm{e}} \sim 1300$ or $\gamma_{\mathrm{e}} \sim 3500$, respectively, for a magnetic field of about $30 \mathrm{G}$. For a cutoff frequency located around $4 \mathrm{keV}$, one obtains $\gamma_{\mathrm{e}} \geq 10^{5}$ for a magnetic field of about $30 \mathrm{G}$.

\subsubsection{The density of relativistic particles}

Yuan et al. (2003) found that the sub-mm radio properties of SgrA* can be explained by pure synchrotron models with densities of the order of $\sim 10^{7.5} \mathrm{~cm}^{-3}$ close to the black hole. Yuan et al. (2004) found that these densities can also explain the X-ray flare fluxes. In this case, high accelerations with $\gamma_{\mathrm{e}} \sim 10^{6}$ of a few percent of the thermal electrons are required. They demonstrated that SSC models need a lower Lorenz factor of about $10^{3}$ and a significantly larger percentage of accelerated particles. Dodds-Eden et al. (2009, 2010) modeled a single, exceptionally bright X-ray flare event using a pure synchrotron description. This illustrates that the need to include as little as possible a SSC contribution to the predicted X-ray flux places stringent constraints on the pure synchrotron model (Dodds-Eden et al. 2010).

However, we know neither the mechanism that accelerates the electrons to relativistic speeds (as stated by most authors) nor the precise population of thermal electrons from which the relativistic particles originate. Typical number densities of $10^{6}-10^{7} \mathrm{~cm}^{3}$ similar to those used for pure synchrotron solutions are derived from radio Faraday rotation. The observed Faraday rotation (Bower et al. 2003; Bower 2003) could take place in a screen at a separation of tens to hundreds of 
Schwarzschild radii from SgrA* (Marrone 2006; Marrone et al. 2006a,b; and discussion by Marrone et al. 2007). However, as discussed in Sect. 5.1.1, the densities close to the mid-plane are not well-defined. Hence, it is also unclear from which population of lower energy electrons the synchrotron and SSC emitting electrons may originate. In the framework of adiabatic expansion, Yusef-Zadeh et al. (2006a) found evidences of densities that may be 100 times higher (see Sect. 6). In the following, we discuss mainly the entire population of relativistic electrons that are responsible for the flare emission.

\subsection{Visualizing SSC solutions}

We adopt the synchrotron self-Compton formalism outlined by Marscher (1983, 2009). We show how the magnetic field strength $B$, the source size $\theta$, the peak flux density $S_{m}$, and the number density $N$ of relativistic particles can be parameterized by the cutoff frequency and spectral index. This allows us to describe the overall variability at (sub-)mm-wavelengths and to constrain the properties of the emission mechanism.

\subsubsection{The formalism}

We assume a synchrotron radio spectrum with a turnover between the optically thick and thin part at a frequency of $v_{m}$ (in $\mathrm{GHz}$ ) and a flux density $\mathrm{S}_{m}$ (in Jy) with an optically thin spectral index $\alpha$

$S_{v} \propto v^{-\alpha}$.

This source component has a bulk velocity of $\beta=v / c$ resulting in a relativistic boosting factor of

$\delta=\Gamma^{-1}[1-\beta \cos \phi]^{-1}$,

were the bulk Lorenz factor is

$\Gamma=\left[1-\beta^{2}\right]^{-1 / 2}$.

In the case of a disk component, the angle $\phi$ is given by the inclination of the disk. The speed is then be a function of the orbital radius $r$ and the fraction of $\beta(r)$ projected onto the line of sight, and reaches its highest value when the component is approaching the observer on its orbit. In the case of a jet component, the angle $\phi$ is given by the angle of the jet to the line of sight. In the framework of disk components, such a jet or fast outflow could be produced by the reconnection of magnetic field lines originating in a disk component and resulting in the acceleration of particles perpendicular to the disk (e.g. Yuan et al. 2009), in analogy to solar mass ejections, were we assume that $\beta(r)=\beta \sim 0.3$ where the disk component is at radii close to or larger than the last stable orbit.

Adopting the formulae given by Marscher (1983, 2009) for the case of the Galactic center, we then find that the SSC X-ray flux density $S_{\mathrm{X}, \mathrm{SSC}}$ (in $\mu \mathrm{Jy}$ ), magnetic field $B$ (in $\mathrm{G}$ ), and column density of relativistic electrons $N_{0}$ (in $\mathrm{cm}^{-3} \mathrm{keV}^{-1}$ ) involved in the emission process are given by

$$
\begin{aligned}
& \left.S_{\mathrm{X}, \mathrm{SSC}}=d(\alpha) \ln \left(\frac{v_{2}}{v_{m}}\right) \theta^{-2(2 \alpha+3)} v_{m}^{-(3 \alpha+5)} S_{m}^{2(\alpha+2)} E_{\mathrm{X}}^{-\alpha} \delta^{-2(\alpha+2)}\right] \\
& B=10^{-5} b(\alpha) \theta^{4} v_{m}^{5} S_{m}^{-2} \delta \\
& N_{0}=n(\alpha) D_{\mathrm{Gpc}}^{-1} \theta^{-(4 \alpha+7)} v_{m}^{-(4 \alpha+5)} S_{m}^{2 \alpha+3} \delta^{-2(\alpha+2)}
\end{aligned}
$$

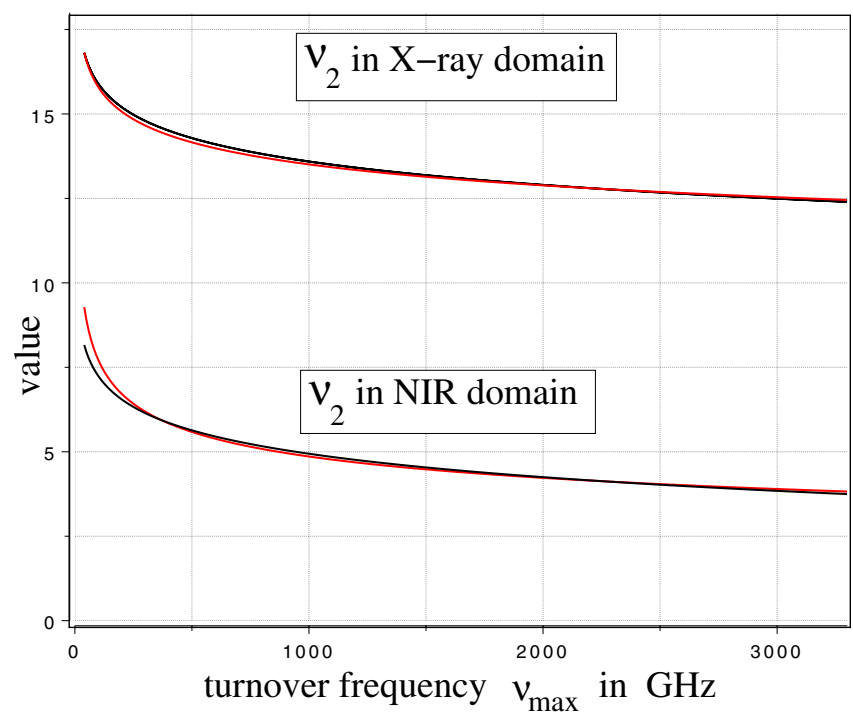

Fig. 2. Approximation of $\ln \left(v_{2} / v_{m}\right)$ in black by the the algebraic Eq. (14) in red.

where $d(\alpha), b(\alpha)$, and $n(\alpha)$ are dimensionless parameters that are all function of $\alpha, D_{\mathrm{Gpc}}$ is the luminosity distance in gigaparsecs and $E_{\mathrm{X}}$ the $\mathrm{X}$-ray photon energy in keV. The underlying relativistic electron distribution is expressed as $N=N_{0} \exp (-p)$ with an electron power-law index $p$. For electron Lorentz factors between $\gamma_{\min }$ and $\gamma_{\max }$, the resulting optically thin electromagnetic spectrum follows a power-law distribution with a spectral index $\alpha=(p-1) / 2$. Here we use $\gamma_{\min }=10$ and $\gamma_{\max }=\gamma_{\mathrm{e}}$.

In the following, we assume an optically thin synchrotron spectrum extrapolated from the near-infrared to the millimeter and sub-mm spectral domain with

$S=\kappa_{1} v^{-\alpha}$,

i.e., $S_{m}=\kappa_{1} v_{m}^{-\alpha}$, and

$\kappa_{1}=S_{\mathrm{NIR}} v_{\mathrm{NIR}}^{\alpha}$

Furthermore, we approximate the natural logarithm in Eq. (9) by the algebraic expression

$\ln (\Re) \sim c_{1} \times \Re^{c_{2}}$

where $\Re=v_{2} / v_{m}$. For models that require synchrotron selfCompton flux density contributions in the NIR or X-ray domain, we assume a cutoff frequency of $v_{2} \sim 140 \mathrm{THz}$ (i.e. at wavelengths just short of the infrared $K$-band), and for models that require dominant synchrotron contributions from the radio to the $\mathrm{X}$-ray domain we assume that $v_{2} \sim 960000 \mathrm{THz}$ (i.e. close to the centers of the typical band-passes of X-ray telescopes such as Chandra and XMM). In Fig. 2, we compare values of $\ln (\Re)$ with the algebraic expression given above. For the two described regimes, a least square fit results in $c_{1}=1.8, c_{2}=0.201$ and $c_{1}=5.36, c_{2}=0.0678$. On average, this relation holds to within better than $2 \%$ for ratios $\Re$ between about a few times $10^{7}$ and less than 50 . This provides the value of $\ln (\Re)$ for turnover frequencies $v_{m}$ ranging from $50 \mathrm{GHz}$ to $3 \mathrm{THz}$. Staying with the same range of turnover frequencies $v_{m}$, a variation in $v_{2}$ by factors of $\sim 2$ is consistent with synchrotron emission in the NIR $J$ - and $H$-band, as well as synchrotron emission over the entire $\mathrm{X}$-ray band-passes of a few $\mathrm{keV}$. 
A. Eckart et al.: Millimeter to X-ray flares from Sagittarius A*
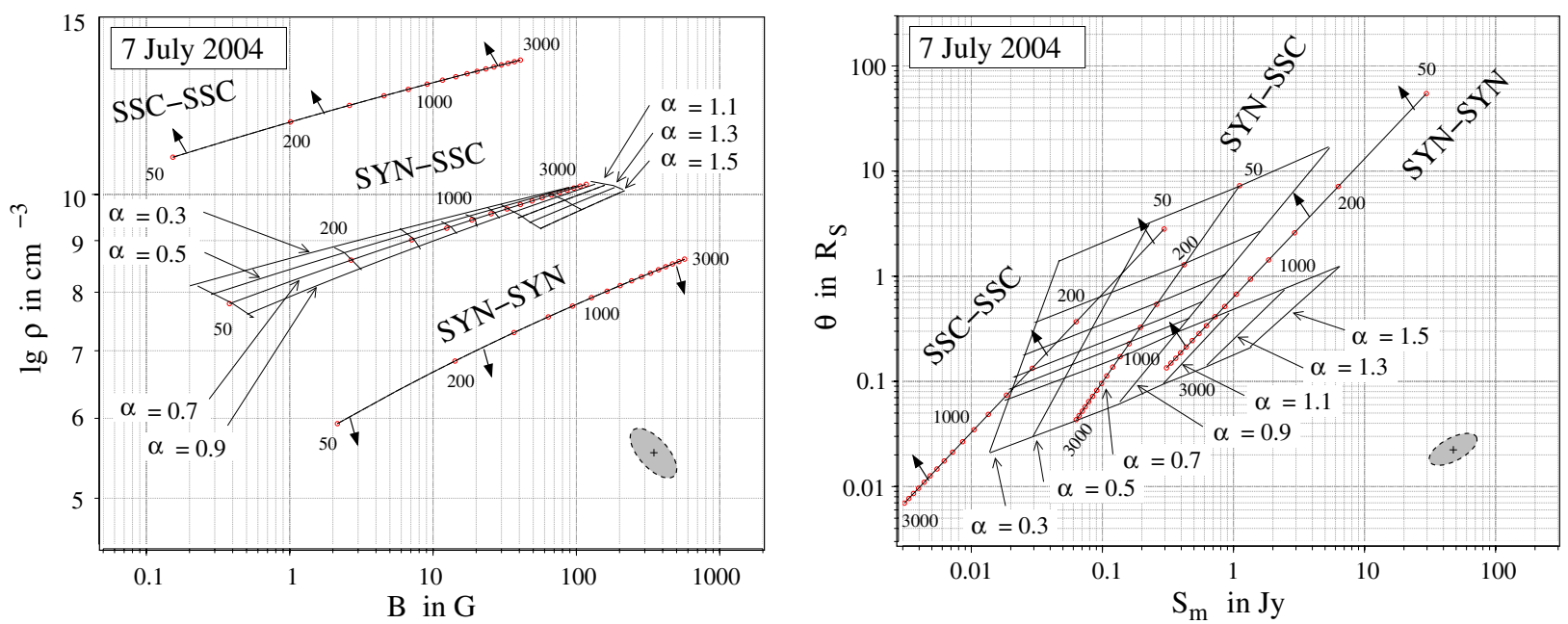

Fig. 3. Synchrotron and self-Compton models for the synchronous NIR and X-ray flare peak of SgrA* observed on 7 July 2004 . The results are shown for the three emission modes (SSC-SSC, SYN-SSC, SYN-SYN) in the particle density/magnetic field plane and in the flux density/source size plane. The grey shaded ellipses and thick arrows are described in the text.

For the self-Compton scattered X-ray flux density, we then find that

$$
\begin{aligned}
S_{\mathrm{X}, \mathrm{SSC}}= & d(\alpha) \ln \left(\frac{v_{2}}{v_{m}}\right) \\
& \times \theta^{-2(2 \alpha+3)} v_{m}^{-(3 \alpha+5)} \kappa_{1}^{2(\alpha+2)} v_{m}^{-2 \alpha(\alpha+2)} E_{\mathrm{X}}^{-\alpha} \delta^{-2(\alpha+2),}
\end{aligned}
$$

resulting in

$1=\kappa_{2}^{\prime} \theta^{-(4 \alpha+6)} v_{m}^{-2 \alpha^{2}-7 \alpha-5-c_{2}}$.

This gives an expression for the source size parameterized in terms of the turnover frequency $v_{m}$ :

$\theta=\kappa_{2} v_{m}^{\zeta_{1}}$

with

$\zeta_{1}=\frac{-2 \alpha^{2}-7 \alpha-5-c_{2}}{4 \alpha+6}$

and

$\kappa_{2}=\left(\kappa_{2}^{\prime}\right)^{\frac{1}{4 \alpha+6}}=\left(S_{\mathrm{X}}^{-1} d(\alpha) c_{1} v_{2}^{c_{2}} \kappa_{1}^{2(\alpha+2)} E_{\mathrm{X}}^{-\alpha} \delta^{-2(\alpha+2)}\right)^{\frac{1}{4 \alpha+6}}$.

For the magnetic field $B$, we find that

$B=10^{-5} b(\alpha) \kappa_{2}^{4} v_{m}^{4 \zeta_{1}} v_{m}^{5} \kappa_{1}^{-2} v_{m}^{+2 \alpha} \delta$,

resulting in an expression for the magnetic field parameterized in the turnover frequency $v_{m}$

$B=\hat{\rho} v_{m}^{\zeta_{2}}$

with

$\zeta_{2}=4 \zeta_{1}+2 \alpha+5$

and

$\hat{\rho}=10^{-5} b(\alpha) \kappa_{2}^{4} \kappa_{1}^{-2} \delta$.

For the number density of relativistic particles, we find that

$$
\begin{aligned}
N_{0}= & n(\alpha) D_{\mathrm{Gpc}}^{-1} \\
& \times \kappa_{2}^{-(4 \alpha+7)} v_{m}^{-\zeta_{1}(4 \alpha+7)} v_{m}^{-(4 \alpha+5)} \kappa_{1}^{2 \alpha+3} v_{m}^{-\alpha(2 \alpha+3)} \delta^{-2(\alpha+2),}
\end{aligned}
$$

resulting in an expression for the number density parameterized in terms of the turnover frequency $v_{m}$ :

$N_{0}=\kappa_{3} v_{m}^{\zeta_{3}}$,

where

$\zeta_{3}=-2 \alpha^{2}-4 \alpha \zeta_{1}-7 \alpha-7 \zeta_{1}-5$

and

$\kappa_{3}=n(\alpha) D_{\mathrm{Gpc}}^{-1} \kappa_{2}^{-(4 \alpha+7)} \kappa_{1}^{2 \alpha+3} \delta^{-2(\alpha+2)}$.

The number density of relativistic particles can then be obtained via

$\rho=m c^{2} \int_{\gamma_{\min }}^{\gamma_{\max }} N(\gamma) \mathrm{d} \gamma=N_{0} \frac{\left(m c^{2}\right)^{-2 \alpha}}{2 \alpha}\left(\gamma_{\min }^{-2 \alpha}-\gamma_{\max }^{-2 \alpha}\right)$,

were $m$ is the electron rest mass. To correct for residual uncertainties in the determination of the source size that results from the above approximation of the logarithm (see Eq. (14)), we adjust the source size $\theta$ such that the observed X-ray flux density is reproduced by the model, using

$S_{\mathrm{X}, \mathrm{SSC}} \propto \theta^{-2(2 \alpha+3)}$

and applying

$\theta_{\text {corrected }}=\theta_{\text {model }}\left(\frac{S_{X, \text { model }}}{S_{X, 0 b e r v e d}}\right)^{\frac{1}{S_{1}}}$

to obtain corrected source-size estimates.

\subsubsection{Parameterized models}

We now use the formalism described above to visualize the parameterized synchrotron and synchrotron self-Compton models of SgrA*. In Fig. 3, we show as a typical example the relativistic electron density $\rho$ as a function of the magnetic field strength $B$ and the source size $\theta$ as a function of the peak flux density $S_{m}$ for the flare observed simultaneously in the NIR and X-ray on 7 July 2004 (Eckart et al. 2006a). We show curves for three cases. The first label SSC-SSC indicates that both the NIR and X-ray flux densities are due to synchrotron self-Compton 
scattered photons and less than $10 \%$ are due to a pure synchrotron contribution. The second label SYN-SYN indicates that the dominant contributions for these two wavelength bands are due to synchrotron radiation and again less than $10 \%$ are due to a SSC contribution. The third label SYN-SSC indicates that the NIR flux density is mainly due to synchrotron and that the X-ray radiation is caused predominantly by a SSC contribution. In the cases of SYN-SYN and SSC-SSC, the spectral indices in the NIR and X-ray domains are the same and given by the respective flux densities in the two bands. In the case of SYN-SSC, the graphs are labeled with the value of the optically thin spectral index of the synchrotron spectrum. The model graphs are only shown as thin solid lines if the NIR and X-ray flux densities are not contaminated by more than $10 \%$ by the synchrotron self-Compton or pure synchrotron contributions, respectively. Mixed models in which both these contaminating contributions are higher than $10 \%$, can be found in regions of the plots located between the SYN-SYN and SSC-SSC graphs. The thick arrows indicate the direction into which the SSC-SSC and SYNSYN model line will move if the synchrotron or self-Compton limit is lowered. The curves shown are dashed if the upper flux density limits obtained in the $8-10 \mu \mathrm{m}$ wavelength regime are violated (Schödel et al. 2007, 2011). In addition, the SSC-SSC, SYN-SYN, and - as an example -the SYN-SSC ( $\alpha=0.7)$ curves are parameterized with the peak synchrotron cutoff frequency $v_{m}$ in $\mathrm{GHz}$ (red dots). The grey shaded ellipses indicate the combined uncertainty in each model point (indicated by the black cross) under the assumption that the speed of the source component varies by $\pm 0.3 c$ centered at $0.3 c$, the inclination varies by $\pm 15^{\circ}$ centered at $45^{\circ}$, and the NIR and X-ray flux densities vary by $20 \%$. These ranges are primarily based on the orbiting spot model. They cover a medium range of inclinations that enabled us to explain the sometimes rapid variations in flux density in terms of boosting. At the same time, the orbiting blobs are allowed to persist at radii that range from the last stable orbit to radii of several $R_{\mathrm{S}}$.

The models in Fig. 3 were calculated for a cutoff frequency range between 50 and $3000 \mathrm{GHz}$ (see Sect. 4). The figure shows that the typical density ranges for the SYN-SYN, the SYN-SSC, and the SSC-SSC models are around $10^{7}, 10^{9}$, and $10^{12} \mathrm{~cm}^{-3}$, respectively. The relativistic electron density typically increases with increasing magnetic field strength, spectral index, and synchrotron cutoff frequency. From SSC-SSC via SYN-SSC to SYN-SYN models and also with decreasing cutoff frequency $v_{m}$ and spectral index $\alpha$, the peak flux density $S_{m}$ and source size $\theta$ both increase.

In Figs. 3-5 we plotted all of the possible solutions parameterized in terms of the spectral index $\alpha$ and the turnover frequency $v_{m}$ that fulfill the observed simultaneous NIR/X-ray flux density measurements. From these figures, we derived the distribution of peak cutoff frequencies (Fig. 6) of the involved synchrotron spectra for the three different cases, SSC-SSC in red, SYN-SSC for $\alpha=+0.7$ in black, and SYN-SYN in blue solid lines. These number distributions represent all possible solutions that give cutoff frequencies that fall between the extreme values derived earlier. The number distributions depend on the boundary conditions as a function of the cutoff frequency $v_{m}$. In Fig. 6 , we show the distributions for three different upper limits of the magnetic field $B$, the synchrotron peak flux density $S_{m}$, and the source size $\theta$, which are consistent with the limits to the $\operatorname{SgrA} *$ source properties discussed in Sect. 4 . While all synchrotron cutoff frequencies up to $3 \mathrm{THz}$ are possible, values of a few hundred $\mathrm{GHz}$ are most likely, especially for smaller and weaker source components. For $\theta<2 R_{\mathrm{S}}, S_{m}<2 \mathrm{Jy}$, and $B<30 \mathrm{G}$, a larger number of NIR/X-ray flares cannot be described by SYN-SSC and SSC-SSC models, and cutoff frequencies of a few hundred $\mathrm{GHz}$ are preferred. For the rejected SSC-SSC and the SYN-SYN models, the violation of the MIR flux density limit does not allow these models to be possible solutions. For the SYN-SSC models, the constraints on the magnetic field, source size, and peak flux density enable us to reject solutions.

\subsection{Modeling the variable spectrum of SgrA*}

The model calculations presented in Figs. 3-5 and in Sect. 4.2.2 can be used to obtain a spectral distribution of the synchrotron self-absorption flux densities. We consider the limits on the magnetic field strength $B$, the synchrotron peak flux density $S_{m}$, the source size $\theta$, and the relativistic particle density $\rho$ as free parameters. Constraining these quantities and combining the model results from all eight flares then gives a frequency-dependent distribution of peak flux densities $S_{m}$ that can be used to describe the variable spectrum of SgrA*. For this, we assume that for the SYN-SSC cases the probability of a spectral index being combined with one of the observed NIR and X-ray flux density pairs is independent of the spectral index values taken out of the +0.3 to +1.5 range of values (in steps of 0.2 ) which we assume are not strongly influenced by synchrotron losses. For the SYN-SSC case, 7 spectral indices, 8 flares, and 16 frequencies between 50 and $3000 \mathrm{GHz}$ (in steps of $200 \mathrm{GHz}$ including $50 \mathrm{GHz}$ ) give a maximum of 896 spectral points. For the SSC-SSC and SYN-SYN cases, this number is 128.

If adiabatic expansion is at work, the flux density contributions of these components will decay towards lower frequencies over times-scales of a few hours depending on their size and expansion speed. If no expansion and no continuous resupply of relativistic electrons takes place, the synchrotron life-time of these particles in the (sub-)mm domain (of a few hours) will determine the flux contribution of the variable source component. Therefore, we can use the $S_{m}$ values as reliable estimates of the flux density measurements at these peak frequencies or just below. The distribution of these fluxes can then be compared to the distribution of the variable fluxes of $\mathrm{SgrA}^{*}$ measured so far in the millimeter and sub-millimeter wavelength domain. To ensure that there are a sufficient number of measurements and model results per bin, we compared our results for three different frequency bands of $75-175 \mathrm{GHz}, 200-375 \mathrm{GHz}, 500-700 \mathrm{GHz}$ for $i=1,2,3$ and $n_{1}=4, n_{2}=11, n_{3}=7$ observed flux values, respectively (see Fig. 7). In each spectral band, we performed our comparison using a figure of merit $\chi^{2}$

$$
\chi^{2}=\frac{1}{n-k} \sum_{i=1}^{3} \frac{n_{i}\left(r_{\mathrm{o}, i}-r_{m, i}\right)^{2}}{\sigma_{i}^{2}}
$$

This quantity probes the ranges over which the observed $\left(r_{\mathrm{O}, i}\right)$ and model predictions of $\left(r_{m, i}\right)$ flux densities vary with respect to the mean measurement uncertainties $\left(\sigma_{i}\right)$, were $n=n_{1}+n_{2}+n_{3}$ and the number of free parameters is $k=4$ (i.e., $B, S_{m}, \theta, \rho$ ).

In Table 5 , we list the upper limits to $B, S_{m}$, and $\theta$, the lower limit to $\log (\rho)$, and the best-fit $\chi^{2}$ value that is obtained using the given limits to the parameters. For the SYN-SSC case, the full range of spectral indices was used (for Fig. 6 we only used the $\alpha=0.7$ ) to allow us to describe the sub-mm flux density variation of $\operatorname{SgrA} *$. With the restricted set of parameters in Table 5, the models closely represent the observed frequency-dependent variability amplitudes. For the SSC-SSC case, the degree of variability at high frequencies is too small to reflect the observations well. For the SYN-SYN case, the degree of variability at low 


\section{Density versus magnetic field}
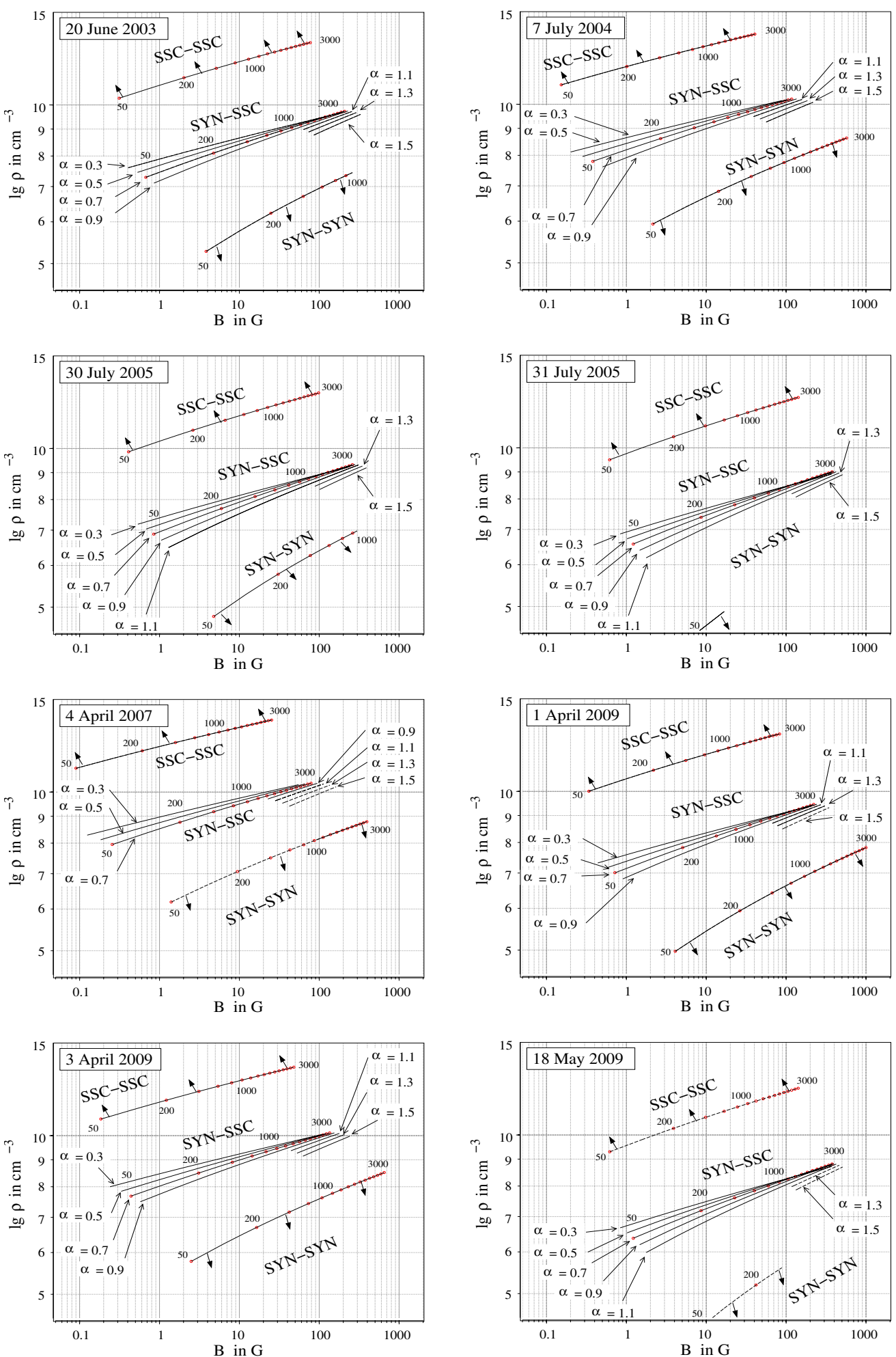

Fig. 4. Summary of possible source models for the three emission modes (SSC-SSC, SYN-SSC, SYN-SYN) in the particle density/ magnetic field plane.

frequencies is much larger that observed and most models are unable to provide an overall description of the SgrA* variability. In summary, under the assumption of a single source component, only the SYN-SSC is the model that is most capable of reproducing the observed $\operatorname{SgrA} *$ variability.
In Fig. 7, we show the observed flux densities of SgrA* taken from the literature (blue) (Zylka et al. 1995; Serabyn et al. 1997; Pierce-Price et al. 2000; Aitken et al. 2000; Marrone et al. 2003) compared to a combined model that consists of the fits given by Falcke et al. (2000) and Marrone et al. (2003) (black 


\section{Source size versus peak flux}
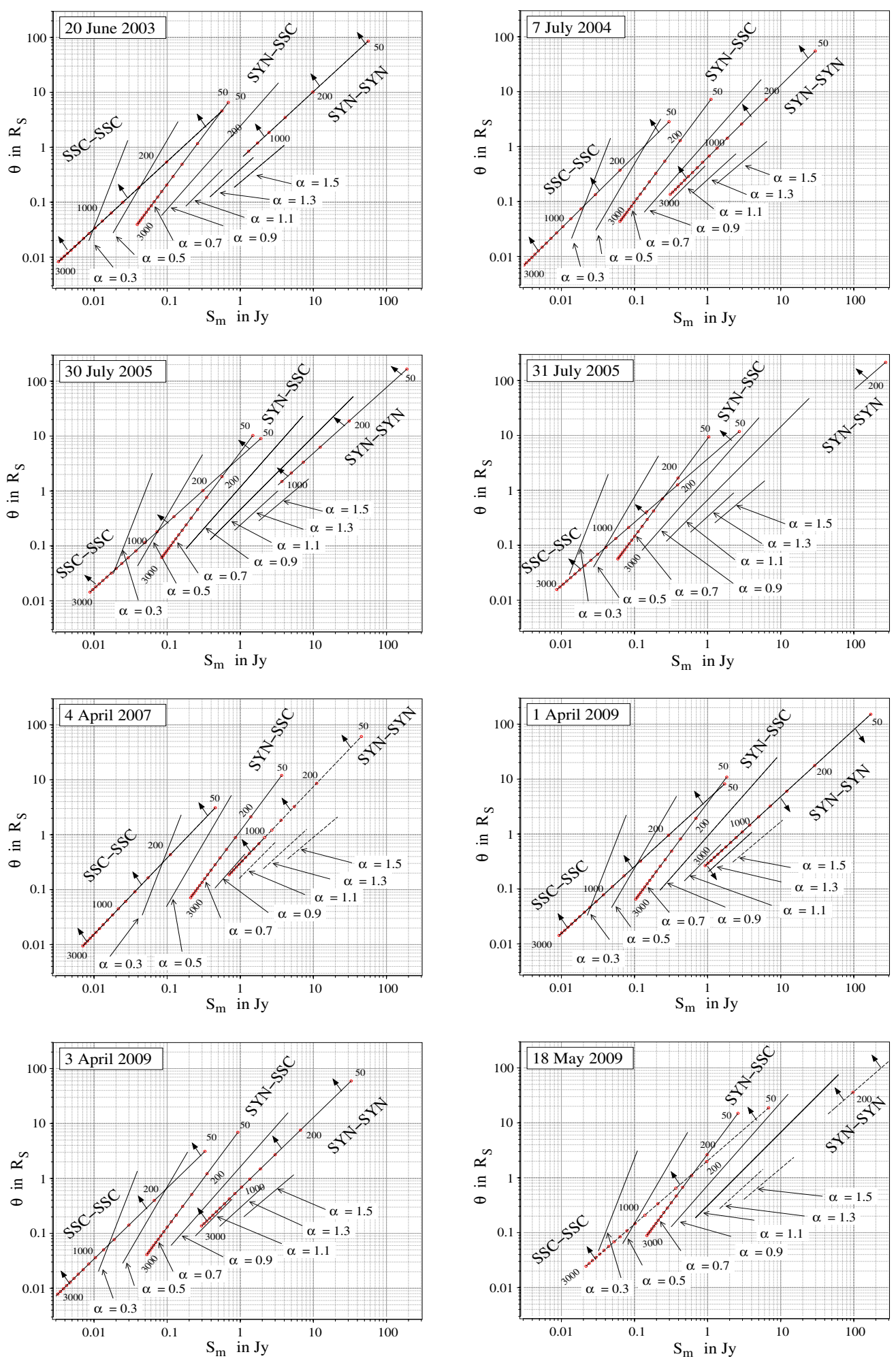

Fig. 5. Summary of possible source models for the three emission modes (SSC-SSC, SYN-SSC, SYN-SYN) in the flux density/source size plane.

line). At frequencies above $100 \mathrm{GHz}$, we selected a model that is representative of the emission contributed through the models presented by Dexter et al. (2010) (black dashed line). This model was chosen to provide a lower bound to the majority of the measured flux density data. The spectrum of synchrotron self-absorption frequencies for the range of models discussed here is shown in red. For the preferred models presented here, the flux-density weighted optically thin spectral index of these self-absorbed source components falls well within the range of observed infrared spectral indices. 

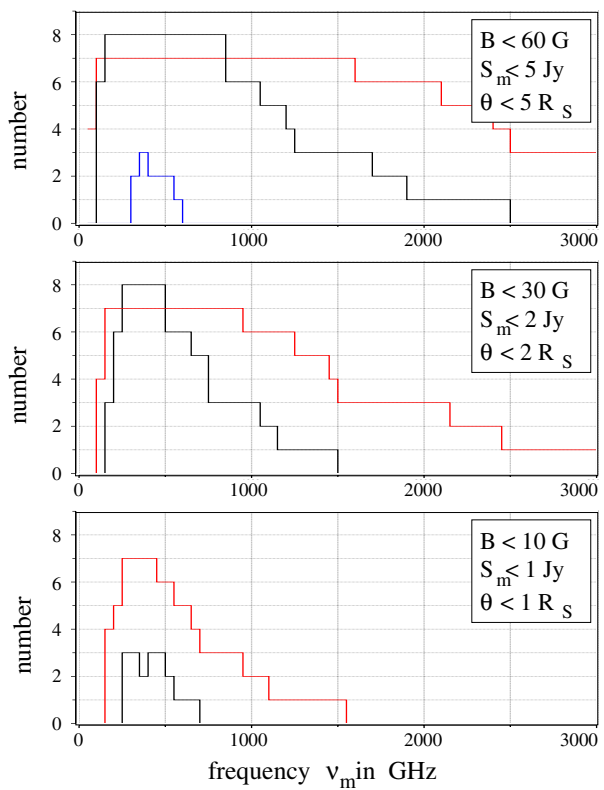

Fig. 6. Distributions of possible synchrotron self-absorption cutoff frequencies obtained from our sample of 8 simultaneous NIR/X-ray flares for the three considered sets of source size $\theta$, flux $S_{m}$, and magnetic field $B$. SSC-SSC in red, SYN-SSC (for $\alpha=+0.7$ ) in black, and $\mathrm{SYN}-\mathrm{SYN}$ in blue.

Table 5. Restricted parameters from modeling the frequency dependent variable flux density of SgrA* for the SYN-SSC, SSC-SSC, and SYN-SYN case.

\begin{tabular}{lccccc}
\hline \hline Case & $B[G]$ & $S_{m}[\mathrm{Jy}]$ & $\theta\left[R_{\mathrm{S}}\right]$ & $\log \rho\left[\mathrm{cm}^{-3}\right]$ & $\chi^{2}$ \\
\hline SYN-SSC & 90 & 5 & 5 & 9.5 & 2.6 \\
SSC-SSC & 90 & 5 & 5 & 9.5 & 7.2 \\
SYN-SYN & 130 & 8 & 2 & 6.8 & 3.6 \\
\hline
\end{tabular}

Notes. We list the upper limits for $B, S_{m}$, and $\theta$ as well as the lower limit for the density $\rho$ and the $\chi^{2}$ values of the model.

\section{Summary and discussion}

In the following we summarize our results and put them into context with other observational and theoretical facts that are essential to understand the emssion processes relevant for SgrA*.

\subsection{The source of variability}

We have demonstrated that modeling the NIR X-ray flare emission using a synchrotron and self-Compton mechanism allows us to explain the observed variability properties of the (sub-)mm spectrum of SgrA*. The constrained source parameters are given in Table 5. In addition, we have found that time-dependent radiative transport models based on relativistic MHD simulations can also explain the observed variability properties of $\mathrm{SgrA}^{*}$ at millimeter wavelengths, although they cannot be linked directly to the observed NIR and X-ray flares. It is therefore likely that both mechansims contribute to the variable emission in the (sub-)mm dmain.

\subsubsection{Sub-mm variability from a magnetized accretion flow}

Dexter et al. (2009) fit time-dependent images of millimeter synchrotron emission from a three-dimensional relativistic MHD code (Fragile et al. 2007) to the VLBI data obtained by Doeleman et al. (2008). Dexter et al. (2010) made use of additional three-dimensional relativistic MHD simulations, including total energy-conserving simulations from McKinney \& Blandford (2009) and simulations of low black hole spins from Fragile et al. (2009). The images were fit to the spectral index measurements from Marrone (2006) as well as to the VLBI data, and two-temperature models of the accretion flow were considered.

In these models, the millimeter emission arises from a compact, hot, magnetized accretion flow close to the black hole. The emission is due to synchrotron radiation from thermal electrons near the mid-plane of the innermost radii of the accretion flow. The vertical particle distribution is well-described by a Gaussian, with a dimensionless scale height of about $0.1-0.3(1 \sigma)$. However, the thickness (hence the mid-plane density) is mostly determined by the initial conditions and energy evolution methods used in the simulations rather than by the physics of the accretion flow. The vertically averaged structure largely consists of $m=1$ type spiral waves. The typical values that describe the physical conditions there are number densities of $n \sim 5 \times 10^{7} \mathrm{~cm}^{3}$, magnetic fields of $B \sim 50 \mathrm{G}$, and electron temperatures of $T_{\mathrm{e}} \sim 5 \times 10^{10} \mathrm{~K}$. These values are consistent with the results obtained by several other authors (e.g. Yuan et al. 2003; Goldston et al. 2005; Moscibrodzka et al. 2009) and reflect results obtained from X-ray measurements for the central few $10 R_{\mathrm{S}}$ (Baganoff et al. 2001, 2003).

The models that most accurately describe the VLBI and spectral observations also exhibit millimeter flares of a few hour duration, half-hour rise time, and a 30-50\% amplitude that all correspond to accretion rate fluctuations driven by the magnetorotational instability. Hence, these models are consistent with the observed millimeter variability of SgrA*. However, since Dexter et al. $(2009,2010)$ concentrate only on modeling the (sub-)millimeter emission, their modeling could not reproduce the observed NIR/X-ray flares and corresponding (sub-)millimeter flares (Eckart et al. 2008; Marrone et al. 2008; Dodds-Eden et al. 2009; Yusef-Zadeh et al. 2008, 2009), which are most likely physically associated. The authors also clearly state that - in the framework of their modeling - it is unclear how their description of variability is linked to the NIR/X-ray flare activity.

\subsubsection{Sub-mm variability and NIR/X-ray flares}

Dexter et al. (2010) pointed out that "non-relativistic simulations are especially inappropriate for modeling the millimeter emission", since the flux "originates in the innermost portion of the accretion flow where relativistic effects are strongest". Therefore we chose to include a moderate amount of relativistic bulk motion similar to that which occurs when either radiating matter is on close orbits around a central black hole or a jet emerges from that region. One result of the analysis that we present here is that a significant portion of the observed variability can be explained via the (sub-)mm flux density contribution expected from the sources responsible for the NIR/X-ray flare emission. The most tightly constrained matching of the variability spectrum of $\operatorname{SgrA}^{*}$ is obtained if the comparison between observed and modeled data is done on the basis of the frequencydependent variability range rather the shape of the variable flux density distribution (Table 5). This may be because the shape of this distribution as a function of frequency has not yet been wellsampled. It may also indicate that - although the overall description of the variable SgrA* spectrum by our model calculations looks reasonable - we may need to modify the assumption that, 

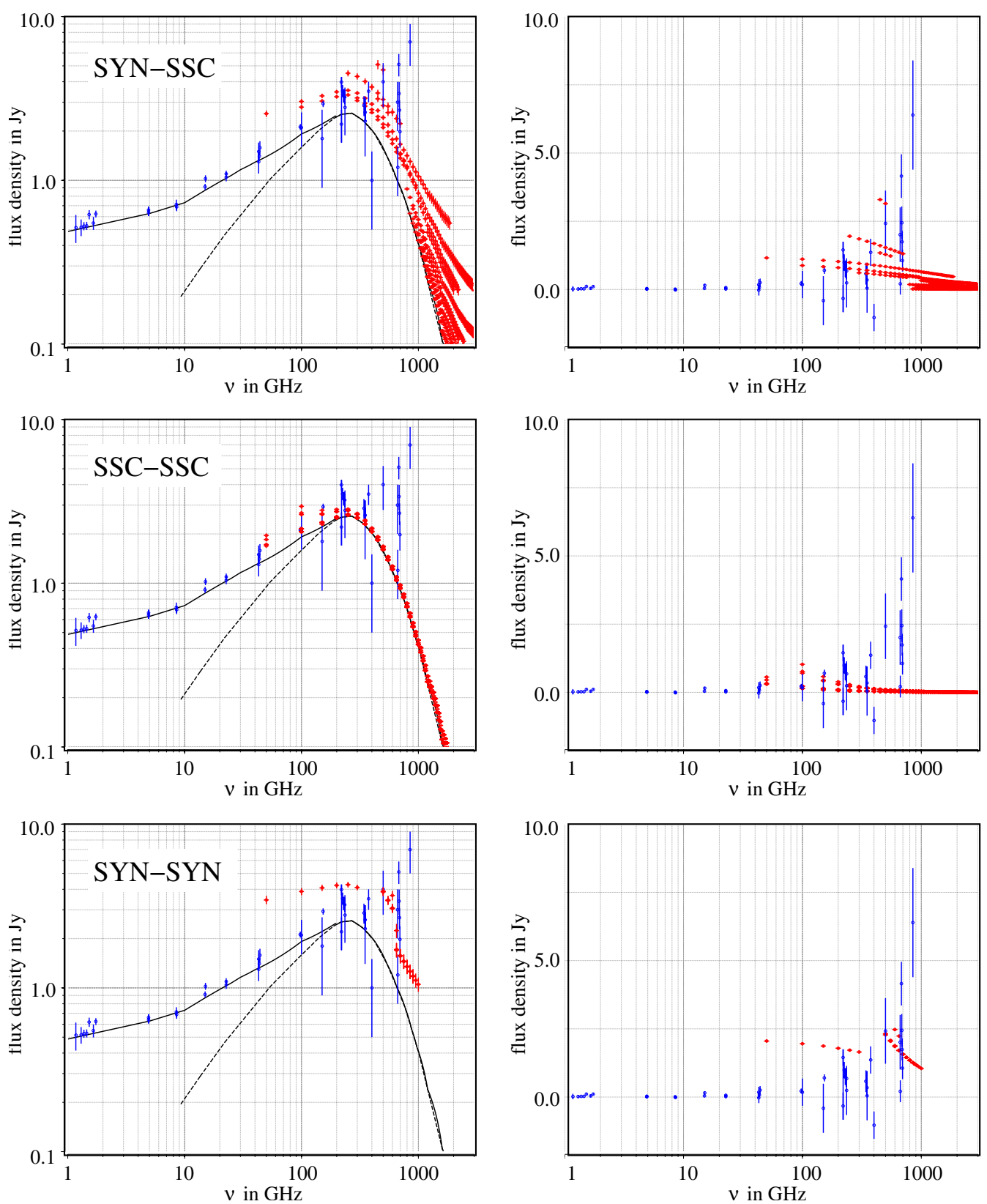

Fig. 7. Observed flux densities of SgrA* taken from the literature (blue) compared to a combined model that consists of the fit given by Falcke et al. (2000), Marrone et al. (2003) (black line), and Dexter et al. (2010) (black dashed line). The spectrum of synchrotron self-absorption frequencies for the range of models discussed here is shown in red. We show results for the SYN-SSC (top), SSC-SSC (middle), and SYN-SYN (bottom) models corresponding to the parameters in Table 5. The SYN-SSC (top) is the preferred model and represents most closely the observed variability of SgrA*. On the left, we show the complete model, on the right we show only the variable flux densities as observed (blue), as well as modeled (red).

for the SYN-SSC models, every NIR/X-ray flux density pair can be combined with every spectral index that is not strongly influenced by synchrotron losses.

The relativistic electron densities in the SYN-SYN models are of the same order as those predicted in the full MHD simulations of the more extended SgrA* accretion flow that extends over the entire central few $10 R_{\mathrm{S}}$ diameter region (e.g. Moscibrodzka et al. 2009). Hence, the density contrast of source components that may result in light-curve modulations caused by orbital motions is very small (if they depend on the density). These models require exceedingly high single electron Lorentz factors with $\gamma_{\mathrm{e}} \sim 10^{6}$. In addition, models with a strong synchrotron contribution at X-ray energies tend to provide strong excess flux contributions owing to the SSC process in this observing band (see discussion in Dodds-Eden 2010).

The SSC-SSC models require high densities of the order of $10^{12} \mathrm{~cm}^{-3}$, however, they typically infer smaller source sizes than the other models. Within a typical flare time-scale of one to two hours the higher density material can expand to the lower density of the accretion flow (Sabha et al. 2010). This may be similar to the process described by Yuan et al. (2009). The authors explain the formation of hot spots in terms of coronal mass ejection comparable to those observed to occur on the Sun. They propose a magnetohydrodynamical model in which by means of low speed shear and turbulence in the disk a flux rope is formed that extends into the disk corona. In a magnetic reconnection 
event, the rope then gets discharged by magnetic compression force leading to mass ejections at relativistic speed.

From Fig. 6, we find that for the SYN-SSC case the bulk of the models have synchrotron turnover frequencies in the range of 300-400 GHz. in agreement with the observations of Marrone (2006) and Marrone et al. (2006a,b). Figure 4 then shows that this corresponds to a median densities of the order of $10^{7.5} \mathrm{~cm}^{-3}$, which compares well with density values in the literature (e.g. Yusef-Zadeh et al. 2006a; Yuan et al. 2003, 2004). However, for models with higher turnover frequencies, higher densities are required (see paramerization in Figs. 3-5). In particular, an appropriate description of the frequency-dependent variability amplitude of SgrA* using the SYN-SSC case (Table 5) results in a $1: 100$ density contrast with respect to the value of $\sim 10^{6.5} \mathrm{~cm}^{-3}$ that is obtained for the accretion flow (e.g. Moscibrodzka et al. 2009). These SYN-SSC models also have moderate Lorentz factors of $\gamma_{\mathrm{e}} \sim 10^{3}$, which can be most easily produced as a high energy tail of the thermal electron distribution than electrons with, e.g., $\gamma_{\mathrm{e}} \sim 10^{6}$.

\subsubsection{MIR flux limit}

As a tendency, we have found that the sub-mm variability can often be more accurately described if one includes models with spectral indices that result in a violation of the MIR flux density limit (see Sect. 4.3). While the $11.88 \mu$ m flux density limit (Dodds-Eden et al. 2010; see comments by Schödel et al. 2011) is included in the uncertainties quoted for model "B I" in Eckart et al. (2008; with or without correction of $\mathrm{A}_{L^{\prime}}$ ), model " $\mathrm{AB} \alpha \phi 3$ " in Eckart et al. (2009) does violate this MIR limit. MIR emission in excess of this $11.88 \mu \mathrm{m}$ limit is also produced by "Model I" for flare A in Trap et al. (2010). In this case, given the spectral index of $\alpha=+2$ (i.e. $p=5$ ), the flux density of $22 \mathrm{Jy}$ at $2 \mathrm{THz}$ results in $138 \mathrm{mJy}$ at a wavelength of $11.88 \mu \mathrm{m}$. This is a factor of 1.6 above the de-reddened $3 \sigma$ upper limit at $11.88 \mu \mathrm{m}$. Even if one takes into account the correction from $A_{L^{\prime}}=1.8$ to $A_{L^{\prime}}=1.23$, one still gets an $11.88 \mu \mathrm{m}$ value of $94 \mathrm{mJy}$ that is above the limit.

Extrapolating from the NIR, an apparent violation of the MIR limit is possible if the spectrum is subject to synchrotron losses. This would imply that the NIR spectra are steeper than the actual optically thin sections of the synchrotron spectra, which are unaffected by the losses. That synchrotron losses are at work is supported by the observed distribution of NIR spectral indices as a function of NIR flare flux density. On the basis of a comparison of histograms of flare peak flux densities in the NIR $K$ s and $H$-band, Bremer et al. (2011) concluded that the distribution of spectral indices as a function of $K$ s-band flux density can be successfully described by an exponential cutoff proportional to $\exp \left[-\left(v / v_{0}\right)^{0.5}\right]$ because of synchrotron losses, were $v_{0}$ is a characteristic cutoff frequency. They find that by varying $v_{0}$ between the NIR and MIR domain and assuming a sub-mm flux density variation of about one Jansky and optically thin spectral indices of $\alpha=+0.7 \pm 0.3$, they can explain the observed spectral properties of SgrA* in the NIR.

Although $\mathrm{SgrA}^{*}$ has not yet been detected at wavelengths of $8 \mu \mathrm{m}$ or longer, in some cases even during NIR flare events (e.g. Schödel et al. 2007, 2011), a dedicated longterm monitoring program to investigate the MIR flux density states of SgrA* still needs to be performed. On the basis of the results of Schödel et al. (2011) a detection of SgrA* at MIR wavelengths may not be possible with the current instrumentation, not even during a typical flare.

\subsection{The $N I R / X$-ray flare profiles}

We have selected in this paper all NIR/X-ray flares that have been observed simultaneously in both bands. With the exception of the NIR flare on 31 July 2005 for which only an upper limit in the X-ray domain is available - they all show a simultaneous (within less than about $10 \mathrm{~min}$ ) near-infrared emission peak (Table 3). At these times, both the flare brightness and the Compton scattering efficiency are high. In general, the flare profiles are more complex. They are not necessarily symmetric with respect to the peak (see the first X-ray flare detection by Baganoff et al. 2001) and significant differences between the NIR and X-ray flare profiles have been reported (e.g., Eckart et al. 2006a; Marrone et al. 2008; Yusef-Zadeh et al. 2008; Sabha et al. 2010; Dodds-Eden et al. 2009, 2010).

A variation in either the source size $\theta$ or the peak flux density $S_{v}$ of the scattered synchrotron component by only a few $10 \%$ will result in the required significant increase or decrease in the SSC scattering efficiency, i.e., following Eq. (9), owing to the scattered SSC X-ray flux density $S_{\text {SSC,X-ray }} \propto S_{m}^{2(\alpha+2)} \theta^{-2(2 \alpha+3)}$, or following Eq. (10), owing to a decrease in the magnetic field $B \propto=\theta^{4} S_{m}^{-2}$, which could be the result of a reconnection process (see e.g. Yuan et al. 2009). Such a variation was already indicated in the first bright flare observed quasi-simultaneously at sub- $\mathrm{mm} / \mathrm{mm}$ wavelengths, and the NIR and X-ray domains (Eckart et al. 2006a, 2009).

In general the discrepances between the flare profiles indicate that the flare process is noisy but that the NIR/ X-ray peak emission and the corresponding ratio represents a characteristic quantity for the flares observed to date.

\subsection{The statistical signature of stochastically distributed adiabatic expansion events}

The new 2009 data set that we present here shows that an adiabatic expansion model can explain the observed flux density variations in the X-ray, NIR, and (sub-) mm-wavelength domains. The need for a third component that peaks around $100 \mathrm{GHz}$ indicates that not all flare components necessarily originate with spectral peaks in the sub-mm band. This indicates that the flare is possibly more structured or that - in general - the process is noisy. In the latter case, this may imply that not all variations at (sub-) mm-wavelengths are necessarily directly related to the NIR/X-ray flares.

In the following, however, we show that even in the presence of a strong noise source that is not directly related physically to the NIR/X-ray flares, adiabatic expansion can be identified (if present) by means of measurements carried out simultaneously at several frequencies (multi-channel measurements). Here we assume that the variable flux density is superimposed onto a flux density offset that can be regarded as constant over the timescales sampled by the lightcurve (see Sect. 3.2). Until now, several events could successfully be described in the framework of adiabatic expansion (e.g., Eckart et al. 2006a, 2008; Yusef-Zadeh et al. 2008, 2009; Trap et al. 2011). This indicates that the relevance of this process to SgrA* has as high probability of being confirmed in the very near future, e.g., through quasi-simultaneous multi-frequency measurements of SgrA* flares, for instance, with advanced multi-channel observing systems like ALMA.

On the basis of observational methodology, we show in the following how statistical support for the adiabatic expansion can be accomplished in the presence of a strong noise source that provides flux density excursions that are unrelated 
to the variability signature of the adiabatically expanding source. Dexter et al. (2010) describe a physical process that is very likely competing in producing variable emission in the (sub-)mm-domain. They point out that a dominant fraction of the radiation is produced by thermal electrons near the mid-plane and close to the inner radius of the accretion disk and that the resulting variability corresponds to flares of $2-3 \mathrm{~h}$ duration and rise times of about $0.5 \mathrm{~h}-$ as observed.

Here we assume that a number of flare events $N_{\mathrm{f}}$ are simultaneously observed in a number of neighboring frequency channels $N_{\mathrm{C}}$ that are several $10 \mathrm{GHz}$ to $100 \mathrm{GHz}$ apart from each other. The length of the observed light curves should be several hours such that the typical time-scales for the adiabatic expansion process can be sampled. In each channel, the uncertainty in detecting an event owing to a disturbing source of variability is $\sigma$. We assume that the noise contribution is strong and that $S_{\text {channel }} / \sigma \sim 1$. We regard such an estimate as realistic since the observed flux density variations can be described either by the model presented by Dexter et al. (2010) or by modeling them using the combination of the SSC process and adiabatic expansion described here.

In addition, we assume that the light curve measurements may oversample the flare events by a factor of $N_{\mathrm{t}}$. Oversampling is only necessary if the variations in the noise source occur at a rate comparable to or shorter than the rate at which the light curve measurements are being conducted (otherwise the noise variations are not be properly sampled). If the variability timescale of the noise source is comparable to that of the adiabatic expansion (as in the case of the noise source described by Dexter et al. 2010), we assume for simplicity that both the competing noise process as well as the adiabatic signal can be described individually in all channels simply by the amplitude and time of the peak flux density excursion in each channel. In this case, we use $N_{\mathrm{t}}=1$.

In the following, we combine the signal (noise and/or adiabatic) by adding the peak amplitudes determined in the individual bands. The combined uncertainty for detecting flare events in these channels is then $\sigma_{\text {comb }}=\sigma \times \sqrt{N_{\mathrm{C}} N_{\mathrm{f}} N_{\mathrm{t}}}$.

The combined signal of a (true) flare event that obeys the amplitude and time relations expected for adiabatic expansion is then $S_{\text {abiab,comb }}=N_{\mathrm{C}} N_{\mathrm{f}} N_{\mathrm{t}} S_{\text {channel }}$. This flux density represents the result of modeling the light curves with only positive flux contributions following the expected time behaviour of an adiabatically expanding source as described in Sect. 3.1. In this modeling, the source size, the flux density, the time of the initial generation of the expanding component, and its linear expansion speed would be free model parameters.

The combined signal for a false flare event in which by chance flux density variations with the required properties are detected in neighboring channels is $S_{\text {false,comb }}=\sigma / \sqrt{N_{\mathrm{C}} N_{\mathrm{f}} N_{\mathrm{t}}}$. This results in a SNR of unity. This would be the result of fitting the flux density excursion in each frequency channel separately. The fit functions should have the shapes expected for an adiabatically expanding source but do not obey the corresponding time dependence and can have positive and negative amplitudes.

The SNR for a true adiabatic combined flare event can then be written as

$S / N=S_{\text {channel }} / \sigma \times \sqrt{N_{\mathrm{C}} N_{\mathrm{f}} N_{\mathrm{t}}}$.

If $N_{\mathrm{C}} \sim N_{\mathrm{t}}$, we can write the SNR as

$S / N \sim S_{\text {channel }} / \sigma N_{\mathrm{C}} \sqrt{N_{\mathrm{f}}}$.

For a significant detection of an adiabatic flare with a SNR of 3-5 and for $N_{\mathrm{t}}=N_{\mathrm{C}}$ and $N_{\mathrm{C}}=2-4$, we then require 3-7 flares. For $N_{\mathrm{t}}=1$, we require up to 13 flares for the same range of signal to noise and frequency channels. While we assume here that the competing noise source is always active, this is not necessarily the case for the adiabatic flare signal. If it is linked to the NIR/X-ray flares it may have an occurrence rate of between 1 and 6 per day since these are the rates at which significant fluctuations are being detected in those frequency domains.

This indicates that if one can observe between half a dozen to a dozen flares over a few neighboring frequency channels and can model them in the framework of adiabatically expanding sources, this can be regarded as statistical support for adiabatic expansion being at work. It is, however, also conceivable that $S_{\text {channel }} / \sigma>1$, in which case a few events that can be modeled as adiabatic expansions would be sufficient to show the statistical significance of this radiation mechanism. The emission mechanism described by Dexter et al. (2010) would then mostly provide the offset flux densities at (sub-)mm wavelengths with contributions of variable flux density on time-scales much longer than the typical few-hour length of a light curve.

\subsection{Millimeter VLBI signature of adiabatic expansion}

Ongoing VLBI observations at $1.3 \mathrm{~mm}$ (mm-VLBI, Doeleman et al. 2008; Fish et al. 2011) may be able to distinguish variabilities caused by magnetic turbulence and adiabatic expansion. In models based on relativistic MHD simulations, the size and shape of the emission region do not change with variations in the total flux. However, an adiabatically expanding blob should cause an observable change in the image morphology and/or FWHM Gaussian size. If the blob is orbiting the central SMBH, or if it is asymmetrically located with respect to the photo-center of the bulk of the mm emission, this may result in a position wander.

From mm-VLBI experiments Reid et al. (2008) have placed limits on such a position wander of SgrA*, measuring the centroid of the SgrA* flux distribution with respect to extragalactic background sources. They found an average centroid wander of $\sim 100 \mu$ as for timescales between 50 and $200 \mathrm{~min}$, with no secular trend. These results are sufficient to begin constraining the viability of the accretion hot-spot model for the radio variability of Sgr A*. The authors can rule out hot spots with orbital radii above $15 \mathrm{GM}_{\mathrm{SgrA} *} / \mathrm{c}^{2}$ which can contribute more than $30 \%$ of the total $7 \mathrm{~mm}$ flux density. Closer or less luminous hot spots remain unconstrained. This limit also applies to off-center source components whose flux density varies significantly owing to adiabatic expansion. An observed NIR/X-ray flare followed shortly by both a total mm-flux variation and a change in either the image size or morphology detected by mm-VLBI, would provide strong evidence of the adiabatic expansion model. The closure phase may also move farther away from zero as the blob contribution increases the asymmetry of the image. Improved monitoring of the centroid position of $\mathrm{SgrA}^{*}$, especially during flares, may be useful to place significant constraints on the existence, kinematics, and morphology of inhomogeneities in the accretion flow of the SMBH at the center of the Milky Way.

\section{Conclusion}

For the bulk of synchrotron and SSC models that have been developed the synchrotron turnover frequencies are in the range of a few hundred GHz. For the pure synchrotron models, this implies densities of relativistic particles of the order of $10^{6.5} \mathrm{~cm}^{-3}$. For models in which the mm- to NIR-flux density depends on the synchrotron and the X-ray flux density because of a significant 
synchrotron self-Compton component, the median densities are an order of magnitude higher. These values are quite comparable to those quoted for the accretion stream toward SgrA* (e.g. Yuan et al. 2003, 2004; Yusef-Zadeh et al. 2006a).

However, our results presented in Fig. 6 indicate that under the stringent boundary conditions of a low self-Compton contribution to the X-ray domain and the low MIR flux density limit only a maximum of three out of eight simultaneously observed flare events can be explained by a pure synchrotron model. The SSC models also appear to be more appropriate if the source components, flux densities, and magnetic fields become smaller. This suggests that if the flares are generated according to a pure synchrotron scenario they are more likely to be associated with the corona of the central object, which also has densities similar to those that can be derived from Faraday rotation measurements. The solutions, however, correspond to uncomfortably high Lorentz factors of $\gamma_{\mathrm{e}} \sim 10^{6}$.

A more robust description that explains all flare events under the above-mentioned boundary conditions is provided by the SYN-SSC models (under the assumption of a single source component responsible for the variable emission). Here we have the situation that rather independent of source size, flux, and magnetic field limits, basically all flare events most likely correspond to synchrotron spectra with turnover frequencies around $300-400 \mathrm{GHz}$, densities around $10^{7} \mathrm{~cm}^{-3}$, and Lorentz factors of $\gamma_{\mathrm{e}} \sim 10^{3}$.

However, for a realistic description of the observed frequency-dependent variability amplitudes of $\mathrm{SgrA}^{*}$ the most general models with high turnover frequencies and two orders of magnitude higher densities are required (see the parameterization in Figs. 3-5). These higher densities are neither unreasonable nor out of reach. In attempting to explain the adiabatic expansion of SgrA* source components, Yusef-Zadeh et al. (2006a) find that for densities of $\sim 6 \times 10^{5} \mathrm{~cm}^{-3}$ an expansion speed of $0.2 \mathrm{c}$ would be expected. Such high expansion velocities are not observed. Much lower speeds require an additional confinement of the source components, resulting in about 100 times higher densities, i.e., from $10^{7} \mathrm{~cm}^{-3}$ to $10^{8} \mathrm{~cm}^{-3}$.

Observationally the physical state of matter in the immediate vicinity of the SgrA* SMBH is rather poorly constrained by existing observations. Only rapid millimeter to X-ray variability (Baganoff et al. 2001; Genzel et al. 2003; Ghez et al. 2004 Mauerhan et al. 2005; Eckart et al. 2004, 2006a, 2008; Yusef-Zadeh et al. 2008, 2009) as well as significant polarization features in the near-infrared (Eckart et al. 2006b; Zamaninasab et al. 2010, 2011) indicate the presence of matter on relativistic orbits close to SgrA*.

However, the radius at which the electrons become relativistic and suppress Faraday rotation within a thermal electron gas is unclear. The linear polarization also needs to be explained in combination with the circular polarization, which may be the result of Faraday conversion from linear to circular (see discussion by Marrone et al. 2007; and Bower et al. 2002; Ruszkowski \& Begelman 2002; Quataert \& Gruzinov 2000). The accretion rate and hence the central density may be much higher than the limits derived from Faraday rotation. In particular, the magnetic field equipartition fraction as well as the bias field strength in the case of magnetic field reversals (as expected for a turbulent flow) are unknown. Variations in the magnetic field structure as well as the field strength with respect to equipartition (Marrone et al. 2007; Igumenshchev et al. 2003) can result in higher densities at distances of only a few Schwarzschild radii from the central SMBH.
Our systematic investigation of the entire current set of simultaneous NIR/X-ray flare events, as well as an attempt to fit the observed variability spectrum of SgrA* in the (sub-)mmdomain may be looked upon as a one of the first steps towards constraining the physical properties of the matter in the immediate vicinity of SgrA*.

Acknowledgements. We thank the anonymous referee for constructive comments. N. Sabha is member of the Bonn Cologne Graduate School (BCGS) for Physics and Astronomy supported by the Deutsche Forschungsgemeinschaft. M. Valencia-S. is member of the International Max-Planck Research School (IMPRS) for Astronomy and Astrophysics at the Universities of Bonn and Cologne supported by the Max Planck Society. F. Baganoff was supported by NASA through Chandra Award Number GO9-0101X and SAO Award Number 2834-MIT-SAO-4018. Part of this work was supported by the COST Action MP0905: Black Holes in a violent Universe and PECS project No. 98040. We are grateful to all members of the NAOS/CONICA, ESO PARANAL, and APEX team. Macarena García-Marín is supported by the German federal department for education and research (BMBF) under the project number 50OS1101. R. Schödel acknowledges support by the Ramón y Cajal programme, by grants AYA2010-17631 and and AYA2009-13036 of the Spanish Ministry of Science and Innovation, and by grant P08-TIC-4075 of the Junta de Andalucía.

\section{References}

Abramowicz, M. A., Lanza, A., Spiegel, E. A., \& Szuszkiewicz, E. 1992, Nature, 356,41

Aitken, D. K., Greaves, J., Chrysostomou, A., et al. 2000, ApJ, 534, L173 Alexander, T. 1997, ASSL, 218, 163

Armitage, P. J., \& Reynolds, C. S. 2003, MNRAS, 341, 1041

Baganoff, F. K., Bautz, M. W., Brandt, W. N., et al. 2001, Nature, 413, 45

Baganoff, F. K., Maeda, Y., Morris, M., et al. 2003, ApJ, 591, 891

Balbus, S. A., \& Hawley, J. F. 1991, ApJ, 376, 214

Balick, B., \& Brown, R. L. 1974, ApJ, 194, 265

Blum, R. D., Sellgren, K., \& Depoy, D. L. 1996, ApJ, 470, 864

Bower, G. C. 2003, Ap\&SS, 288, 69

Bower, G. C., Falcke, H., Sault, R. J., \& Backer, D. C. 2002, ApJ, 571, 843

Bower, G. C., Wright, M. C. H., Falcke, H., \& Backer, D. C. 2003, ApJ, 588, 331

Broderick, A. E., \& Loeb, A. 2006, MNRAS, 367, 905

Bremer, M., Witzel, G., Eckart, A., et al. 2011, A\&A, 532, A26

Buchholz, R. M., Schödel, R., \& Eckart, A. 2009, A\&A, 499, 483

Dexter, J., Agol, E., \& Fragile, P. C. 2009, ApJ, 703, L142

Dexter, J., Agol, E., Fragile, P. C., \& McKinney, J. C. 2010, ApJ, 717, 1092

Diolaiti, E., Bendinelli, O., Bonaccini, D., et al. 2000, A\&AS, 147, 335

Do, T., Ghez, A. M., Morris, M. R., et al. 2009a, ApJ, 691, 1021

Doeleman, S. S. Weintroub, J., Rogers, A. E. E., et al. 2008, Nature, 455, 78

Doeleman, S. S., Fish, V. L., Broderick, A. E., Loeb, A., \& Rogers, A. E. E. 2009, ApJ, 695, 59

Dodds-Eden, K., Porquet, D., Trap, G., et al. 2009, ApJ, 698, 676

Dodds-Eden, K., Sharma, P., Quataert, E., et al. 2010, ApJ, 725, 450

Eckart, A., Genzel, R., Ott, T., \& Schödel, R. 2002, MNRAS, 331, 917

Eckart, A., Baganoff, F. K., Morris, M., et al. 2004, A\&A, 427, 1

Eckart, A., Baganoff, F. K., Schödel, R., et al. 2006a, A\&A, 450, 535

Eckart, A., Schödel, R., Meyer, L., et al. 2006b, A\&A, 455, 1

Eckart, A., Baganoff, F. K., Zamaninasab, M., et al. 2008, A\&A, 479, 625

Eckart, A., Baganoff, F. K., Morris, M. R., et al. 2009, A\&A, 500, 935

Eckart, A., Zamaninasab, M., Sabha, N., et al. 2011, The Galactic center: a

Window to the Nuclear Environment of Disk Galaxies. Proceedings of a workshop held at Shanghai, China on October 19-23, 2009, ed. M. R. Morris, Q. Daniel Wang, \& Feng Yuan (San Francisco: ASP), 294

Eisenhauer, F., Genzel, R., Alexander, T., et al. 2005, ApJ, 628, 246

Fish, V. L., Doeleman, S. S., Beaudoin, C., et al. 2011, ApJ, 727, L36

Falanga, M., Melia, F., Tagger, M., Goldwurm, A., \& Belanger, G. 2007, ApJ, 662, L15

Falcke, H., \& Markoff, S. 2000, A\&A, 362, 113

Falcke, H., Goss, W. M., Matsuo, H., et al. 1998, ApJ, 499, 731

Fragile, P. C. 2009, ApJ, 706, L246

Garcia-Marin, M., Eckart, A., Weiss, A., et al. 2011a, ASPC, 439, 315

García-Marín, M., Eckart, A., Weiss, A., et al. 2011b, ApJ, 738, 158

Genzel, T., Eckart, A., Ott, T., \& Eisenhauer 1997, MNRAS, 291, 219

Ghez, A. M., Duchéne, G., Matthews, K., et al. 2003, ApJ, 586, L127

Ghez, A. M., Wright, S. A., Matthews, K., et al. 2004, ApJ, 601, 159

Gillessen, S., Eisenhauer, F., Quataert, E., et al. 2006, ApJ, 640, L163

Goldston, J. E., Quataert, E., \& Igumenshchev, I. V. 2005, ApJ, 621, 785

Hornstein, S. D., Matthews, K., Ghez, A. M., et al. 2007, ApJ, 667, 900

Igumenshchev, I. V., Narayan, R., \& Abramowicz, M. A. 2003, ApJ, 592, 1042 
Jones, T. W., O'dell, S. L., \& Stein, W. A. 1974a, ApJ, 188, 353 Jones, T. W., O'dell, S. L., \& Stein, W. A. 1974b, ApJ, 192, 261 Karas, V., Dovciak, M., Eckart, A., \& Meyer, L. 2007, Proceedings of the Workshop on the Black Holes and Neutron Stars, ed. S. Hledik, \& Z. Stuchlik, 19-21 September (Silesian University, Opava), 99

Kunneriath, D., Witzel, G., Eckart, A., et al. 2010, A\&A, 517, A46

Li, J., Shen, Z.-Q., Miyazaki, A., et al. 2009, ApJ, 700, L417

Lucy, L. B. 1974, AJ, 79, L745

McKinney, J. C., \& Blandford, R. D. 2009, MNRAS, 394, L126

Macquart, J.-P., \& Bower, G. C. 2006, ApJ, 641, 302

Markoff, S. 2005, ApJ, 618, L103

Markoff, S., Bower, G. C., \& Falcke, H. 2007, MNRAS, 379, 1519

Marrone, D. P. 2006, Ph.D. Thesis, Harvard Univ.

Marrone, D. P., Moran, J. M., Zhao, J.-H., \& Rao, R. 2006a, ApJ, 640, 308

Marrone, D. P., Moran, J. M., Zhao, J.-H., \& Rao, R. 2006b, JPhCS, 54, 354

Marrone, D. P., Moran, J. M., Zhao, J.-H., \& Rao, R. 2007, ApJ, 654, L57

Marrone, D. P., Baganoff, F. K., Morris, M., et al. 2008, ApJ, 682, 373

Marscher, A. P. 1983, ApJ, 264, 296

Marscher, A. P. 2009, in The Jet Paradigm - From Microquasars to Quasars, Lect. Notes Phys., ed. T. Belloni, 794

Mauerhan, et al. 2005, ApJ, 623, L25

Melia, F., \& Falcke, H. 2001, ARA\&A, 39, 309

Moscibrodzka, M., Gammie, C. F., Dolence, J. C., Shiokawa, H., \& Leung, P. K. 2009, ApJ, 706, 497

Narayan, R., Yi, I., \& Mahadevan, R. 1995, Nature, 374, 623

Pauliny-Toth, I. I. K., \& Kellermann, K. I. 1966, ApJ, 146, 634

Pecháček, T., Karas, V., \& Czerny, B. 2008, A\&A, 487, 815

Pierce-Price, D., Richer, J. S., Greaves, J. S., et al. 2000, ApJ, 545, L121

Poutanen, J., \& Fabian, A. C. 1999, MNRAS, 306, L31

Quataert, E., \& Gruzinov, A. 2000, ApJ, 545, 842

Reid, M.J., Broderick, A. E., Loeb, A., Honma, M., \& Brunthaler, A. 2008, ApJ, 682,1041
Ruszkowski, M., \& Begelman, M. C. 2002, ApJ, 573, 485

Sabha, N., Witzel, G., Eckart, A., et al. 2010, A\&A, 512, A2

Schnittman, J. D., Krolik, J. H., \& Hawley, J. F. 2006, ApJ, 651, 1031

Shcherbakov, R. V., \& Baganoff, F. K. 2010, ApJ, 716, 504

Schödel, R., Ott, T., Genzel, R., et al. 2002, Nature, 419, 694

Schödel, R., Eckart, A., Alexander, T., et al. 2007, A\&A, 469, 125

Schödel, R., Najarro, F., Muzic, K., \& Eckart, A. 2010, A\&A, 511, A18

Schödel, R., Morris, M. R., Muzic, K., et al. 2011, A\&A, 532, A83

Scoville, N. Z., Stolovy, S. R., Rieke, M., Christopher, M., \& Yusef-Zadeh, F. 2003, ApJ, 594, 294

Serabyn, E., Carlstrom, J., Lay, O., et al. 1997, ApJ, 490, L77

Singal, A. K. 2009, ApJ, 703, L109

Siringo, G., Weiss, A., Kreysa, E., et al. 2007, ESO Messenger, 129, 2

Trap, G., Goldwurm, A., Dodds-Eden, K., et al. 2011, A\&A, 528, A140

van der Laan, H. 1966, Nature, 211, 1131

Weiss, A., Kovacs, A., Guesten, R., et al. 2008, A\&A, 490, 77

Weisskopf, M. C., Brinkman, B., Canizares, C., et al. 2002, PASP, 114, 1

Yuan, F., Quataert, E., \& Narayan, R. 2003, ApJ, 598, 301

Yuan, F., Quataert, E., \& Narayan, R. 2004, ApJ, 606, 894

Yuan, F., Lin, J., Wu, K., Ho, \& Luis C. 2008, MNRAS, 395, 2183

Yusef-Zadeh, F., et al. 2006a, ApJ, 650, 189

Yusef-Zadeh, F., et al. 2006b, ApJ, 644, 198

Yusef-Zadeh, F., Wardle, M., Heinke, C., et al. 2008, ApJ, 682, 361

Yusef-Zadeh, F., Bushouse, H., Wardle, M., et al. 2009, ApJ, 706, 348

Zamaninasab, M., Eckart, A., Witzel, G., et al. 2010, A\&A, 510, A3

Zamaninasab, M., Eckart, A., Dovciak, M., et al. 2011, MNRAS, 413, 322

Zhao, J.-H., Young, K. H., Herrnstein, R. M., et al. 2003, ApJ, 586, L29

Zycki, P. T. 2002, MNRAS, 333, 800

Zylka, R., Mezger, P. G., Ward-Thompson, D., Duschl, W. J., \& Lesch, H. 1995, A\&A, 297, 83 